\title{
Relative Hamiltonian for Faithful Normal States of a von Neumann Algebra
}

By

\author{
Huzihiro ARAKI
}

\begin{abstract}
Let $\Psi$ be a cyclic and separating vector for a von Neumann algebra $\mathfrak{M}$ and $\Delta_{\Psi}$ be its modular operator. For any elements $Q_{1}, \ldots, Q_{n}$ in $\mathfrak{M}$ and complex numbers $z_{1}, \ldots, z_{n}$ such that $\operatorname{Re} z_{j} \geqq 0$ and $\Sigma \operatorname{Re} z_{j} \leqq 1 / 2, \Psi$ is shown to be in the domain of $\Delta_{\Psi}^{z} Q_{1} \ldots \Delta_{\Psi}^{z} n Q_{n}$ and $\left\|\Delta_{\Psi_{1}^{1}}^{z} Q_{1} \ldots \Delta_{\Psi}^{z} n Q_{n} \Psi\right\| \leqq\left\|Q_{1}\right\| \ldots\left\|Q_{n}\right\|\|\Psi\|$.

A selfadjoint operator $h=h(\varphi / \psi) \in \mathfrak{M}$ is called a Hamiltonian of a faithful normal state $\varphi$ of $\mathfrak{M}$ relative to another faithful state $\psi$ of $\mathfrak{M}$ if vectors $\xi_{\varphi}$ and $\xi_{\psi}$ representing $\varphi$ and $\psi$ (in the canonical cone $V_{\Psi}^{1 / 4}$ ) is related by

$$
\xi_{\varphi}=\sum_{n=0}^{\infty}(-1)^{n} \int_{0}^{1 / 2} \mathrm{~d} t_{1} \int_{0}^{t_{1}} \mathrm{~d} t_{2} \cdots \int_{0}^{t_{n-1}} \mathrm{~d} t_{n} \Delta \xi_{\psi}^{t_{n}} h \Delta_{\xi_{\psi}}^{t_{n-1}-t} n h \ldots \Delta \Delta_{\xi_{\psi}}^{t_{1}-t_{2}} h \xi_{\psi} .
$$

The operator

$$
u_{t}^{\varphi \psi}=\sum_{n=0}^{\infty}(-i)^{n} \int_{0}^{t} \mathrm{~d} t_{1} \int_{0}^{t_{1}} \mathrm{~d} t_{2} \ldots \int_{0}^{t_{n-1}} \mathrm{~d} t_{n} \sigma_{t_{n}}^{\psi}(h) \sigma_{t_{n-1}}^{\psi}(h) \ldots \sigma_{t_{1}}^{\psi}(h)
$$

is shown to be an intertwining unitary operator between modular automorphisms $\sigma_{t}^{\psi}$ and $\sigma_{t}^{\varphi}$ for states $\psi$ and $\varphi$ :

$$
u_{t}^{\varphi \psi} \sigma_{t}^{\psi}(x)=\sigma_{t}^{\varphi}(x) u_{t}^{\varphi \psi}, x \in \mathfrak{M} .
$$

The relative hamiltonian $\mathrm{h}(\varphi / \phi)$ is unique for given states $\varphi$ and $\psi$. It exists and satisfies $\log l_{1} \geqq-\mathrm{h}(\varphi / \psi) \geqq \log l_{2}$ if $l_{1}^{1 / 2} \xi_{\psi} \geqq \xi_{\varphi} \geqq l_{2}^{1 / 2} \xi_{\psi}$, where $\Phi_{1} \geqq \Phi_{2}$ means that $\Phi_{1}-\Phi_{2}$ is in the canonical cone $V_{\Psi}^{1 / 4}$. In particular, if $l_{1} \psi \geqq \varphi \geqq l_{2} \psi$, then $\mathrm{h}(\varphi / \psi)$ exists and satisfies the above inequality.

The modular operators $\Delta_{\xi_{\varphi}}$ and $\Delta_{\xi_{\psi}}$ are related by

$$
\log \Delta_{\xi_{\psi}}-\log \Delta_{\xi_{\varphi}}=\mathrm{h}(\varphi / \psi)-J \mathrm{~h}(\varphi / \psi) J
$$

where $J$ is the common modular conjugation operator for $\xi_{\varphi}$ and $\xi_{\psi}$. The chain rule $\mathrm{h}\left(\varphi_{1} / \varphi_{2}\right)+\mathrm{h}\left(\varphi_{2} / \varphi_{3}\right)=\mathrm{h}\left(\varphi_{1} / \varphi_{3}\right)$ is satisfied.
\end{abstract}

\section{§1. Introduction}

Let $\mathfrak{M}$ be a $*$-algebra of finite matrices and $\varphi_{0}$ be a faithful tracial state on $\mathfrak{M}$. Then every positive linear functional $\varphi$ on $\mathfrak{M}$ is uniquely Received December 7, 1972. 
represented by a positive element $\rho_{\varphi}$ of $\mathfrak{M}$ by

$$
\varphi(x)=\varphi_{0}\left(\rho_{\varphi} x\right), \quad x \in \mathfrak{M} .
$$

The corresponding modular automorphism $\sigma_{t}^{\varphi}$ is given by

$$
\sigma_{t}^{\varphi}(x)=\rho_{\varphi}^{i t} x \rho_{\varphi}^{-i t}, \quad x \in \mathfrak{M} .
$$

The $L_{2}$-norm $\|x\|_{2} \equiv \varphi_{0}\left(x^{*} x\right)^{1 / 2}$ makes $\mathfrak{M}$ a Hilbert space which we shall write $\mathfrak{S}$. The vector corresponding to $1 \in \mathfrak{M}$ is written as $\xi_{0}$.

To each $\varphi \in \mathfrak{M}_{*}^{+}$, there corresponds a canonical vector $\xi_{\varphi}=\rho_{\varphi}^{1 / 2} \xi_{0}$ such that the expectation functional $\omega_{\xi_{\varphi}}$ by the vector $\xi_{\varphi}$ is $\varphi$. The set of $\xi_{\varphi}$ is a selfdual convex cone $\mathfrak{M}^{+} \xi_{0}$ in $\mathfrak{E}$, which has been denoted as $V_{\xi_{0}}$ or $V \frac{1 / 4}{\xi_{0}}$ in [2]. The modular conjugation operator $J$ for $\xi_{\varphi}$ is conmon for all faithful $\varphi \in \mathfrak{M}_{*}^{+}$and is given by

$$
J x \xi_{0}=x^{*} \xi_{0} .
$$

The modular operator $\Delta_{\xi_{\varphi}}$ for $\xi_{\varphi}$ is given by

$$
\Delta_{\xi_{\varphi}} x \xi_{0}=\rho_{\varphi} x \rho_{\varphi}^{-1} \xi_{0} .
$$

In statistical mechanics, $h_{\varphi}=-\log \rho_{\varphi}$ for a faithful $\varphi \in \mathfrak{M}_{*}^{+}$is called a Hamiltonian, $\sigma_{-t}^{\varphi}$ is called a time translation automorphism and $\varphi$ is called the Gibbs state for this Hamiltonian (with an inverse temperature $\beta=1$ ). We shall be concerned with a Hamiltonian of a faithful state $\varphi$ relative to another faithful state $\psi$ defined by

$$
\mathrm{h}(\varphi / \psi)=h_{\varphi}-h_{\psi}
$$

The original $h_{\varphi}$ is $\mathrm{h}\left(\varphi / \varphi_{0}\right)$. Since a tracial state is not available for a purely infinite von Neumann algebra, we are forced to work with the relative Hamiltonian in the general case.

From (1.2), we immediately see that the unitary operator

$$
u_{t}^{\varphi \phi}=\rho_{\phi}^{i t} \rho_{\phi}^{-i t}
$$

intertwines modular automorphisms of $\varphi$ and $\psi$ :

$$
u_{t}^{\varphi \psi} \sigma_{t}^{\phi}(x)=\sigma_{t}^{\varphi}(x) u_{t}^{\varphi \psi}, \quad x \in \mathfrak{M} .
$$

The operator in (1.6) has the following perturbation expansion 


$$
\begin{aligned}
u_{t}^{\varphi \psi} & =\sum_{n=0}^{\infty}(-i)^{n} \int_{0}^{t} \mathrm{~d} t_{1} \int_{0}^{t_{1}} \mathrm{~d} t_{2} \ldots \int_{0}^{t_{n-1}} \mathrm{~d} t_{n} \sigma_{t_{n}}^{\psi}(h) \ldots \sigma_{t_{1}}^{\psi}(h) \\
& =\sum_{n=0}^{\infty}(-i)^{n} \int_{0}^{t} \mathrm{~d} t_{1} \int_{0}^{t_{1}} \mathrm{~d} t_{2} \ldots \int_{0}^{t_{n-1}} \mathrm{~d} t_{n} \sigma_{t_{1}}^{\varphi}(h) \ldots \sigma_{t_{n}}^{\varphi}(h)
\end{aligned}
$$

where $h=\mathrm{h}(\varphi / \psi)$.

For two faithful states $\psi$ and $\varphi$ in $\mathfrak{M}_{*}^{+}$, there exists a unique $\mathrm{A}(\varphi / \psi)$ $\in \mathfrak{M}$ such that $\mathrm{A}(\varphi / \psi) \xi_{w}=\xi_{\varphi}$. It is the Radon-Nikodym derivative satisfying the chain rule [2] and is given by

$$
\mathrm{A}(\varphi / \psi)=\rho_{\varphi}^{1 / 2} \rho_{\text {小 }}^{-1 / 2},
$$

which has the following perturbation expansion:

$$
\xi_{\varphi}=\mathrm{A}(\varphi / \psi) \xi_{\psi}=\sum_{n=0}^{\infty}(-1)^{n} \int \ldots \int \mathrm{d} \lambda_{1} \ldots \mathrm{d} \lambda_{n} \Delta \Delta_{\psi}^{\lambda_{\psi}} h \ldots \Delta \Delta_{\psi}^{\lambda n} h \xi_{\psi}
$$

where the integration is over the following simplex

$$
\bar{I}_{n}^{1 / 2} \equiv\left\{\left(\lambda_{1}, \ldots, \lambda_{n}\right) ; \lambda_{1} \geqq 0, \ldots, \lambda_{n} \geqq 0,1 / 2 \geqq \lambda_{1}+\cdots+\lambda_{n}\right\} .
$$

These expansion formulas are of the same form as the covariant perturbation expansion in an interaction picture used in quantum field theory and has been discussed in [3] in the Banach algebra context.

The purpose of the present paper is to show that the relative hamiltonian $\mathrm{h}(\varphi / \psi)$ exists for a certain class of the pair $\varphi, \phi$ in $\mathfrak{M}_{*}^{+}$, it is unique and it satisfies (1.7), (1.10), the chain rule

$$
\mathrm{h}\left(\varphi_{1} / \varphi_{2}\right)+\mathrm{h}\left(\varphi_{2} / \varphi_{3}\right)=\mathrm{h}\left(\varphi_{1} / \varphi_{3}\right)
$$

and

$$
\log \Delta_{\xi_{\varphi}}-\log \Delta_{\xi_{\psi}}=\mathrm{h}(\psi / \varphi)-j_{\Psi}(h(\psi / \varphi))
$$

\section{§2. Multi-variable three-line theorem}

The following is an immediate generalization of the three-line theorem of Doetsch to the case of many complex variables.

Theorem 2.1. Let $\mathrm{f}(z)$ be a function of $n$ complex variables $z=$ 
$\left(z_{1} \ldots z_{n}\right)$ satisfying the following two conditions:

(i) $\mathrm{f}(z)$ is holomorphic in the tube

$$
\mathrm{T}(B)=\{z ; \operatorname{Im} z \in B\}
$$

where $B$ is an open convex set in $R^{n}$.

(ii) $\mathrm{f}(z)$ is continuous and bounded in the closure $\overline{\mathrm{T}}(B)=\mathrm{T}(\bar{B})$.

Let

$$
\mathrm{g}(y)=\sup _{x}|\mathrm{f}(x+i y)|, \quad y \in \bar{B}
$$

Then $\log g(y)$ is a convex function of $y \in \bar{B}$.

Proof. We have to prove the inequality

$$
\log g\left(\lambda y_{a}+(1-\lambda) y_{b}\right) \leqq \lambda \log g\left(y_{a}\right)+(1-\lambda) \log g\left(y_{b}\right)
$$

for $0 \leqq \lambda \leqq 1, y_{a} \in \bar{B}$ and $y_{b} \in \bar{B}$.

If $\mathrm{g}\left(y_{0}\right)=0$ at one point $y_{0}$, then $\mathrm{f}\left(x+i y_{0}\right)=0$ for all $x \in R^{n}$ and hence $\mathrm{f}(z)=0$ identically (by the edge of wedge theorem if $y_{0} \in \partial B$ ). In this case, $\log \mathrm{g}(y)=-\infty$ for all $y \in \bar{B}$ and (2.3) holds. We now assume that $\mathrm{f}(z) \not 0$ and hence $\mathrm{g}(y) \neq 0$.

For each $y_{a}$ and $y_{b}$, we may restrict our attention to a compact convex subset of $\bar{B}$ containing $y_{a}, y_{b}$ and a non-empty interior. Hence we may assume that $\bar{B}$ is compact without loss of generality.

First consider the case where $\lambda y_{a}+(1-\lambda) y_{b} \in B$ for $0<\lambda<1$. Consider a function of one complex variable $z_{0}$ :

$$
\mathrm{f}_{x}\left(z_{0}\right)=\mathrm{f}\left(i\left\{z_{0} y_{a}+\left(1-z_{0}\right) y_{b}\right\}+x\right), x \in R^{n} .
$$

If $\operatorname{Re} z_{0} \in(0,1)$, then $\mathrm{f}_{x}\left(z_{0}\right)$ is holomorphic by the tentative assumption, continuous and bounded in the closure. By Doetsch's three-line theorem, $\log \sup _{\sigma \in R}\left|f_{x}(\lambda+i \sigma)\right|$ is a convex function of $\lambda$ in $[0,1]$. Since the supremum of a family of convex functions is convex, we have the convexity of

$$
\sup _{x \in R^{n}} \log \sup _{\sigma}\left|f_{x}(\lambda+i \sigma)\right|=\log g\left(\lambda y_{a}+(1-\lambda) y_{b}\right)
$$

in $\lambda$ and hence (2.3).

For general points $y_{a}$ and $y_{b}$ in $\bar{B}$, we consider a sequence of points 
$y_{a}^{n}$ and $y_{b}^{n}$ such that $\lim y_{a}^{n}=y_{a}, \lim y_{b}^{n}=y_{b}$ and $\lambda y_{a}^{n}+(1-\lambda) y_{b}^{n} \in B$ for $0<\lambda<1$ and all $n$. Instead of $\mathrm{f}$, we first consider the function

$$
\mathrm{f}_{\beta}(z)=\mathrm{f}(z) \exp \left(-\beta \sum z_{j}^{2}\right) . \quad(\beta>0 .)
$$

For compact $\bar{B}, \mathrm{f}_{\beta}(z)$ tends uniformly to 0 as $z$ tends to $\infty$ in $T(\bar{B})$. Let

$$
\mathrm{g}_{\beta}(y)=\sup _{x}\left|\mathrm{f}_{\beta}(x+i y)\right|
$$

Then it is continuous in $y \in \bar{B}$. By what we have already proved we have

$$
\log \mathrm{g}_{\beta}\left(\lambda y_{a}^{n}+(1-\lambda) y_{b}^{n}\right) \leqq \lambda \log \mathrm{g}_{\beta}\left(y_{a}^{n}\right)+(1-\lambda) \log \mathrm{g}_{\beta}\left(y_{b}^{n}\right) \text {. }
$$

By continuity, we have

$$
\log \mathrm{g}_{\beta}\left(\lambda y_{a}+(1-\lambda) y_{b}\right) \leqq \lambda \log \mathrm{g}_{\beta}\left(y_{a}\right)+(1-\lambda) \log \mathrm{g}_{\beta}\left(y_{b}\right)
$$

We can now complete the proof by showing that

$$
\lim _{\beta \rightarrow+0} g_{\beta}(y)=g(y)
$$

for each $y \in \bar{B}$. First we have

$$
\left|\exp \left(-\beta \sum z_{j}^{2}\right)\right| \leqq \exp \left(\beta \sup _{y \in B} \sum y_{j}^{2}\right) \rightarrow 1
$$

as $\beta \rightarrow 0$. ( $\bar{B}$ is assumed to be compact at this stage of the proof.) Hence for sufficiently small $\beta$,

$$
\mathrm{g}_{\beta}(y) \leqq \mathrm{g}(y)+\varepsilon,
$$

for any given $\varepsilon>0$. There exists $x$ such that

$$
|\mathrm{f}(x+i y)| \geqq \mathrm{g}(y)-\varepsilon / 2 .
$$

For this $x$, we have $\left|\mathrm{f}_{\beta}(x+i y)\right| \geqq \mathrm{g}(y)-\varepsilon$ for sufficiently small $\beta$. Hence

$$
\mathrm{g}(y)-\varepsilon \leqq \mathrm{g}_{\beta}(y) \leqq \mathrm{g}(y)+\varepsilon
$$

for sufficiently small $\beta$ for any given $\varepsilon>0$.

Q.E.D.

Remark. The convexity of $\log g(y)$ implies the convexity of $g(y)$. 
By considering $\exp f(z)$ instead of $f(z)$, we also obtain the convexity of $\sup _{x} \operatorname{Re} \mathrm{f}(x+i y)$.

Corollary 2.2. Let $B$ in Theorem 2.1 be a simplex

$$
B=I_{n}^{a} \equiv\left\{y ; y_{1}>0, \ldots, y_{n}>0, a>y_{1}+\cdots+y_{n}\right\}, a>0 .
$$

Then $g(y)$ takes its maximum value in $\bar{B}$ at one of $(n+1)$ extremal points of $\bar{B}: \lambda^{0} \equiv(0, \ldots, 0), \lambda^{1} \equiv(a, 0, \ldots, 0), \lambda^{2} \equiv(0, a, 0, \ldots, 0), \ldots, \lambda^{n} \equiv(0, \ldots, 0, a)$.

Proof. Since $\log \mathrm{g}(y)$ is convex, it takes its maximum value in a compact convex set $\bar{B}$ at one of its extremal points.

Q.E.D.

\section{§3. Multiple KMS Property}

Theorem 3.1. Let $\Psi$ be a cyclic and separating vector with a modular operator $\Delta_{\Psi}$. It is in the domain of

$$
\Delta_{\Psi}^{i z_{1}} Q_{1} \Delta_{\Psi}^{i z_{2}} Q_{2} \ldots \Delta_{\Psi}^{i z_{n}} Q_{n} \equiv \mathrm{A}(z)
$$

if $Q_{k} \in \mathfrak{M}, k=1, \ldots, n$, and $z=\left(z_{1} \ldots z_{n}\right) \in \mathrm{T}\left(-I_{n}^{1 / 2}\right)$. The vector valued function $\mathrm{A}(z) \Psi$ of $z$ is holomorphic in the tube $\mathrm{T}\left(-I_{n}^{1 / 2}\right)$, strongly continuous and uniformly bounded in its closure $\overline{\mathrm{T}}\left(-I_{n}^{1 / 2}\right)$ :

$$
\|\mathrm{A}(z) \Psi\| \leqq\|\Psi\|\left\|Q_{1}\right\| \cdots\left\|Q_{n}\right\|
$$

Proof. We prove Theorem by induction on $n$. When $n=0$, there is nothing to prove. Assume that Theorem is true for $n<m$ except that the strong continuity is temporarily replaced by the weak continuity and consider the case $n=m$.

Let $\Phi \in \mathrm{D}\left(\Delta_{\Psi}^{1 / 2}\right)$. Then $\Phi \in \mathrm{D}\left(\Delta_{\Psi}^{i z_{1}}\right)$ for $\operatorname{Im} z_{1} \in[-1 / 2,0]$. Consider

$$
\begin{aligned}
& \mathrm{f}(z)=\left(\Phi_{1}, \Phi_{2}\right), \\
& \Phi_{1}=Q_{1} \Delta_{\Psi}^{i z_{2}} Q_{2} \Delta_{\Psi}^{i z_{3}} \ldots \Delta_{\Psi}^{i z_{m}} Q_{m} \Psi \\
& \Phi_{2}=\Delta_{\bar{\Psi}} \overline{\bar{T}}^{i \bar{z}_{1}} \Phi
\end{aligned}
$$

By Lemma 4 of $[2]$, we have 


$$
\left\|\Phi_{2}\right\|^{2} \leqq\left\|\Delta_{\Psi}^{1 / 2} \Phi\right\|^{2}+\|\Phi\|^{2}
$$

Hence $\Phi_{2}$ is a uniformly bounded and strongly continuous function of $z_{1}$ for $z_{1} \in \overline{\mathrm{T}}\left(-I_{1}^{1 / 2}\right)$. By inductive assumption, $\Phi_{1}$ is a uniformly bounded and weakly continuous function of $\left(z_{2}, \ldots, z_{m}\right) \in \overline{\mathrm{T}}\left(-I_{m-1}^{1 / 2}\right)$. Hence $\mathrm{f}(z)$ is a uniformly bounded continuous function of $z=\left(z_{1}, \ldots, z_{n}\right)$ in $\overline{\mathrm{T}}\left(-I_{1}^{1 / 2}\right)$ $\times \overline{\mathrm{T}}\left(-I_{m-1}^{1 / 2}\right)$, which contains $\overline{\mathrm{T}}\left(-I_{m}^{1 / 2}\right)$. Since $\Phi_{2}$ is holomorphic for $z_{1} \in \mathrm{T}\left(-I_{1}^{1 / 2}\right)$ and $\Phi_{1}$ is holomorphic for $\left(z_{2}, \ldots, z_{m}\right) \in \mathrm{T}\left(-I_{m}^{1 / 2}\right), \mathrm{f}(z)$ is holomorphic in $\mathrm{T}\left(-I_{1}^{1 / 2}\right) \times \mathrm{T}\left(-I_{m-1}^{1 / 2}\right)$ by Hartogs' theorem.

We now consider $|\mathrm{f}(z)|$ when $\operatorname{Im} z$ is at one of extremal points of $-\bar{I}_{m}^{1 / 2}$. We have

$$
|\mathrm{f}(x)| \leqq\left\|\Phi|\||\Psi|\|| Q_{1}\right\| \cdots\left\|Q_{m}\right\|, \quad x \in R
$$

If $\operatorname{Im} z_{j}=0$ except for $j=k$ and $\operatorname{Im} z_{k}=-1 / 2$, we have

$$
\begin{gathered}
\mathrm{f}(z)=\left(Q_{1}\left(x_{1}\right) \cdots Q_{k-1}\left(x_{1}+\cdots+x_{k-1}\right) \Delta \frac{1 / 2}{\Psi} Q_{k}\left(x_{1}+\cdots+x_{k}\right) \cdots\right. \\
\left.Q_{m}\left(x_{1}+\cdots+x_{m}\right) \Psi, \Phi\right) \\
=\left(Q_{1}\left(x_{1}\right) \cdots Q_{k-1}\left(x_{1}+\cdots+x_{k-1}\right) J_{\Psi} Q_{m}^{*}\left(x_{1}+\cdots+x_{m}\right) \cdots\right. \\
\left.Q_{k}^{*}\left(x_{1}+\cdots+x_{k}\right) \Psi, \Phi\right)
\end{gathered}
$$

where $x_{l}=\operatorname{Re} z_{l}$ and $Q(t)=\Delta_{\Psi}^{i t} Q \Delta_{\Psi}^{-i t}$. Hence we have

$$
|\mathrm{f}(z)| \leqq|| \Phi|||\Psi||| \mid Q_{1}\|\cdots\| Q_{m} \| .
$$

for all $z \in \overline{\mathrm{T}}\left(-I_{m}^{1 / 2}\right)$. By Riesz theorem, there exists a vector $\Phi(z)$ such that $\mathrm{f}(z)=(\Phi(z), \Phi)$ and $\|\Phi(z)\| \leqq\|\Psi\|\left\|Q_{1}\right\| \cdots\left\|Q_{m}\right\|$. Hence $\Phi_{1}$ is in the domain of $\Delta^{i z_{1}}$ and (3.2) holds for $n=m$.

Since $\mathrm{f}(z)$ is holomorphic in $\mathrm{T}\left(-I_{m}^{1 / 2}\right)$ and continuous in, $\overline{\mathrm{T}}\left(-I_{m}^{1 / 2}\right)$, the uniform boundedness implies that $\Phi(z)=\mathrm{A}(z) \Psi$ is weakly continuous in $\overline{\mathrm{T}}\left(-I_{m}^{1 / 2}\right)$ and weakly holomorphic in $\mathrm{T}\left(-I_{m}^{1 / 2}\right)$. Since the weak and strong holomorphy are the same due to Cauchy integral formula for polycircles, we have the desired properties for $n=m$.

To show the strong continuity from the weak continuity, it is enough to show the continuity of the norm. This follows from the next Theorem.

Q.E.D.

The following Theorem states a multiple KMS property and has been 
derived in somewhat different but equivalent context in [1], except for (4).

Theorem 3.2. There exists a function $\mathrm{F}(z)$ of $z=\left(z_{1}, \ldots, z_{n}\right)$ for $a$ given cyclic and separating vector $\Psi$ and operators $Q_{1}, \ldots, Q_{n+1} \in \mathfrak{M}$ such that

(1) $\mathrm{F}(z)$ is holomorphic in $z \in \mathrm{T}\left(-I_{n}^{1}\right)$,

(2) $\mathrm{F}(z)$ is continuous in $z \in \overline{\mathrm{T}}\left(-I_{n}^{1}\right)$,

(3) $\mathrm{F}(z)$ is uniformly bounded in $z \in \overline{\mathrm{T}}\left(-I_{n}^{1}\right)$ :

$$
|\mathrm{F}(z)| \leqq\|\Psi\|^{2}\left\|Q_{1}\right\| \cdots\left\|Q_{n+1}\right\|
$$

(4) if $z \in \overline{\mathrm{T}}\left(-I_{n}^{1}\right)$ and

$$
\operatorname{Im}\left(z_{1}+\cdots+z_{k-1}\right) \geqq-1 / 2, \quad \operatorname{Im}\left(z_{k}+\cdots+z_{n}\right) \geqq-1 / 2,
$$

then $\mathrm{F}(z)=\left(\Phi_{1}, \Phi_{2}\right)$ where

$$
\begin{aligned}
& \Phi_{1}=\Delta_{\Psi}^{\alpha} Q_{k} \Delta_{\Psi}^{i z{ }_{k-1}} Q_{k-1} \ldots \Delta_{\Psi}^{i z} Q_{1} \Psi, \\
& \Phi_{2}=\Delta_{\bar{\Psi}^{-\alpha-i \bar{z}_{k}}} Q_{k+1}^{*} \Delta_{\bar{\Psi}}^{-i \bar{z}_{k+1}} Q_{k+2}^{*} \ldots \Delta_{\bar{\Psi}}^{-i \bar{z}_{n}} Q_{n+1}^{*} \Psi,
\end{aligned}
$$

$\alpha$ is any non-negative real number satisfying

$$
\begin{aligned}
& -\operatorname{Im} z_{k} \geqq \alpha \geqq 0, \\
& 1 / 2+\operatorname{Im}\left(z_{1}+\cdots+z_{k-1}\right) \geqq \alpha \geqq-1 / 2-\operatorname{Im}\left(z_{k}+\cdots+z_{n}\right),
\end{aligned}
$$

(5) if $\operatorname{Im} z=0$, then

$$
\mathrm{F}(z)=\omega_{\Psi}\left(Q_{n+1} Q_{n}\left(x_{n}\right) Q_{n-1}\left(x_{n-1}+x_{n}\right) \ldots Q_{1}\left(x_{1}+\cdots+x_{n}\right)\right),
$$

(6) if $\operatorname{Im} z_{k}=0$ except for $\operatorname{Im} z_{j}=-1$, then

$$
\mathrm{F}(z)=\omega_{\Psi}\left(Q_{j}\left(x_{j}+\cdots+x_{n}\right) \ldots Q_{1}\left(x_{1}+\cdots+x_{n}\right) Q_{n+1} \ldots Q_{j+1}\left(x_{j+1}+\cdots+x_{n}\right)\right),
$$

where $x_{j}=\operatorname{Re} z_{j}$ and $Q(t)=\Delta_{\Psi}^{i t} Q \Delta_{\Psi}^{-i t}$.

Proof. Let $Q_{j, \beta}=Q_{j}\left(f_{\beta}^{G}\right)$ where $f_{\beta}^{G}$ is given by (3.11) of [2] and the notation $Q(f)$ is given by (3.7) of $[2] . \quad Q_{j, \beta}(t)$ has an analytic continuation $Q_{j, \beta}(z) \in \mathfrak{M}$ for any complex number $z$ and 


$$
\begin{aligned}
\mathrm{F}^{\beta}(z) & \equiv\left(Q_{n+1, \beta} \Delta_{\Psi}^{i z_{n}} Q_{n, \beta} \ldots \Delta_{\Psi}^{i z_{1}} Q_{1, \beta} \Psi, \Psi\right) \\
& =\left(Q_{n+1, \beta} Q_{n, \beta}\left(z_{n}\right) \ldots Q_{1, \beta}\left(z_{n}+\cdots+z_{1}\right) \Psi, \Psi\right)
\end{aligned}
$$

is an entire function of $z$. By Theorem 2.1, $\left|\mathrm{F}^{\beta}(z)-\mathrm{F}^{\beta^{\prime}}(z)\right|$ for $z \in$ $\mathrm{T}\left(-\bar{I}_{n}^{1}\right)$ is bounded by the maximum of $\left|\mathrm{F}^{\beta}\left(x-i \lambda^{(j)}\right)-\mathrm{F}^{\beta^{\prime}}\left(x-i \lambda^{(j)}\right)\right|$ for $j=0,1, \ldots, n$ and real $x$ where $\lambda^{(j)}$ is as in Corollary 2.2 in which we set $a=1$.

By usual KMS condition, we have

$$
\begin{array}{r}
\mathrm{F}^{\beta}\left(x-i \lambda^{(j)}\right)=\left(Q_{j, \beta}\left(x_{j}+\cdots+x_{n}\right) \ldots Q_{1, \beta}\left(x_{1}+\cdots+x_{n}\right) Q_{n+1, \beta} \cdots\right. \\
\left.Q_{j+1, \beta}\left(x_{j+1}+\cdots+x_{n}\right) \Psi, \Psi\right) .
\end{array}
$$

Since $\left\|Q_{j, \beta}\right\| \leqq\left\|Q_{j}\right\|, \lim _{\beta \rightarrow 0} Q_{j, \beta}=Q_{j}$ and $\lim _{\beta \rightarrow 0} Q_{j, \beta}^{*}=Q_{j}^{*},\left|\mathrm{~F}^{\beta}-\mathrm{F}^{\beta^{\prime}}\right|$ converges to zero uniformly in $z$ in any compact subset of $\mathrm{T}\left(-\bar{I}_{n}^{1}\right)$. Let $\mathrm{F}(z)=$ $\lim _{\beta \rightarrow 0} \mathrm{~F}^{\beta}(z)$. As the uniform limit of a continuous and holomorphic function, $F(z)$ is continuous on $\mathrm{T}\left(-\bar{I}_{n}^{1}\right)$ and holomorphic in $\mathrm{T}\left(-I_{n}^{1}\right)$. Since $\left|\mathrm{F}^{\beta}(z)\right| \leqq\left(\prod_{j}\left\|Q_{j, \beta}\right\|\right)\|\Psi\|^{2} \leqq\left(\prod_{j}\left\|Q_{j}\right\|\right)\|\Psi\|^{2}$, (3) is also satisfied. Hence $\mathrm{F}(z)$ so constructed satisfies (1), (2), (3), (5) and (6). It remains to prove (4).

Consider

$$
\begin{aligned}
& \mathrm{f}\left(z_{1} \ldots z_{n+1}\right)=\mathrm{F}\left(z_{1} \ldots z_{k-1}, z_{k}+z_{k+1}, \ldots z_{n+1}\right)-\left(\Psi_{1}, \Psi_{2}\right) \\
& \Psi_{1}=\Delta_{\Psi}^{i z_{k}} Q_{k} \Delta_{\Psi}^{i z_{k-1}} Q_{k-1} \ldots \Delta_{\Psi}^{i z_{1}} Q_{1} \Psi \\
& \Psi_{2}=\Delta_{\Psi}^{-i z_{k+1}} Q_{k+1}^{*} \ldots \Delta_{\Psi}^{-i \bar{z}_{n+1}} Q_{n+1}^{*} \Psi
\end{aligned}
$$

in the domain

$$
\begin{aligned}
& \left(z_{1} \ldots z_{k}\right) \in \mathrm{T}\left(-I_{k}^{1 / 2}\right),\left(z_{k+1} \ldots z_{n+1}\right) \in \mathrm{T}\left(-I_{n-k+1}^{1 / 2}\right), \\
& \left(z_{1} \ldots z_{k}+z_{k+1} \ldots z_{n+1}\right) \in \mathrm{T}\left(-I_{n}^{1}\right)
\end{aligned}
$$

$\mathrm{f}(z)$ is holomorphic in this domain.

If $\operatorname{Im}\left(z_{1}+\cdots+z_{n+1}\right) \geqq-1 / 2$, then

$$
\left(\Psi_{1}, \Psi_{2}\right)=\left(Q_{n+1} \Delta_{\Psi}^{i z_{n+1}} \ldots Q_{k+1} \Delta_{\Psi}^{i\left(z_{k+1}+z_{k}\right)} Q_{k} \ldots \Delta_{\Psi}^{i z_{1}} Q_{1} \Psi, \Psi\right)
$$

By the weak continuity of Theorem $3.1, \mathrm{f}(z)$ is continuous in $z \in \overline{\mathrm{T}}\left(-I_{n+1}^{1 / 2}\right)$. At $\operatorname{Im} z=0$, we have $\mathrm{f}(z)=0$. Hence $\mathrm{f}(z)=0$ identically by edge of 
wedge theorem. This proves (4) for $z \in \overline{\mathrm{T}}\left(-I_{n}^{1}\right)$ satisfying $-\alpha+\operatorname{Im}\left(z_{1}+\cdots+z_{k-1}\right)>-1 / 2, \operatorname{Im}\left(z_{k}+\cdots+z_{n}\right)+\alpha>-1 / 2$.

For a fixed $z_{1} \ldots z_{k-1}$, we obtain (4) for $z \in \overline{\mathrm{T}}\left(-I_{n}^{1}\right)$ satisfying

$$
-\alpha+\operatorname{Im}\left(z_{1}+\cdots+z_{k-1}\right)>-1 / 2, \quad \operatorname{Im}\left(z_{k}+\cdots+z_{n}\right)+\alpha \geqq-1 / 2
$$

by the weak continuity. For fixed $z_{k} \ldots z_{n+1}$, we then obtain (4) for $z \in \overline{\mathrm{T}}\left(-I_{n}^{1}\right)$ satisfying

$$
-\alpha+\operatorname{Im}\left(z_{1}+\cdots+z_{k-1}\right) \geqq-1 / 2, \operatorname{Im}\left(z_{k}+\cdots+z_{n}\right)+\alpha \geqq-1 / 2
$$

by weak continuity again.

Q.E.D.

\section{§4. Relative Hamiltonian}

Proposition 4.1. Let $h \in \mathfrak{M}, h^{*}=h$ and $\Psi$ be a cyclic and separating vector. Then

$$
\Psi(h)=\sum_{n=0}^{\infty} \int \cdots \int \mathrm{d} t_{1} \cdots \mathrm{d} t_{n} \Delta_{\Psi}^{t_{n}} h \Delta_{\Psi}^{t_{n-1}} h \ldots \Delta_{\Psi}^{t_{1}} h \Psi
$$

converges absolutely and uniformly over bounded $h$, where the integrations are over the region

$$
\bar{I}_{n}^{1 / 2}=\left\{\left(t_{1} \ldots t_{n}\right) ; t_{1} \geqq 0, \ldots, t_{n} \geqq 0, t_{1}+\cdots+t_{n} \leqq 1 / 2\right\} .
$$

$\Psi(h)$ defined by (4.1) is in $V_{\Psi}$, namely the closure of $\Delta_{\Psi}^{1 / 4} \mathfrak{M}^{+} \Psi[2]$. If a sequence $h_{n}$ converges to $h$ strongly, then $\Psi\left(h_{n}\right)$ converges to $\Psi(h)$ strongly.

Proof. By Theorem 3.1, we have

$$
\left\|\Delta_{\Psi}^{t_{n}} h \Delta_{\Psi}^{t_{n-1}} h \cdots \Delta_{\Psi}^{t_{1}} h \Psi\right\| \leqq\|h\|^{n}\|\Psi\|
$$

Hence (4.1) is dominated by $\Sigma(n !)^{-1}\|h\|^{n}\|\Psi\|=e^{\|h\|}\|\Psi\|$ and hence converges absolutely and uniformly over a bounded set of $h$.

Since converging sequence is uniformly bounded and multiplication is continuous on a bounded set, we have

$$
\lim \sigma_{t_{1}}^{\psi}\left(h_{n}\right) \ldots \sigma_{t_{m}}^{\psi}\left(h_{n}\right) \Psi=\sigma_{t_{1}}^{\psi}(h) \ldots \sigma_{t_{m}}^{\psi}(h) \Psi
$$


for each $t_{1} \ldots t_{m}$. By Lebesgue dominated convergence theorem, we have the strong convergence (i.e. weak convergence plus the convergence of norm):

$$
\lim _{n} h_{n}\left(f_{1}\right) \ldots h_{n}\left(f_{m}\right) \Psi=h\left(f_{1}\right) \ldots h\left(f_{m}\right) \Psi
$$

for any $L_{1}$ functions $f_{1} \ldots f_{m}$, where

$$
h_{n}(f)=\int \sigma_{t}^{\psi}\left(h_{n}\right) f(t) \mathrm{d} t, \quad h(f)=\int \sigma_{t}^{\psi}(h) f(t) \mathrm{d} t .
$$

We shall use $f_{\beta}^{G}$ given by (3.11) of [2].

If $\mathrm{F}\left(z_{1} \ldots z_{n}\right)$ is a vector valued function holomorphic in $\left\{z ;\left|z_{j}-z_{j}^{0}\right| \leqq \delta\right\}$ and bounded by $\mathrm{A}$, then for $\left|z_{j}-z_{j}^{0}\right| \leqq \delta / 2$, we have

$$
\begin{aligned}
\left\|\left(\mathrm{d} / \mathrm{d} z_{j}\right) \mathrm{F}\right\| & =\left\|(2 \pi i)^{-1} \int_{\left|z-z_{j}^{0}\right|=\delta}\left(z-z_{j}\right)^{-2} \mathrm{~F}\left(z_{1} \ldots z_{j-1} z z_{j+1} \ldots z_{n}\right) \mathrm{d} z\right\| \\
& \leqq 4 A \delta^{-1}
\end{aligned}
$$

Hence $\left\|F(z)-F\left(z^{\prime}\right)\right\| \leqq 4 A \delta^{-1}\left|z-z^{\prime}\right|$ for $\left|z_{j}-z_{j}^{0}\right| \leqq \delta / 2,\left|z_{j}^{\prime}-z_{j}^{0}\right| \leqq \delta / 2$. Since

$$
\Delta_{\Psi}^{s_{1}} \sigma_{t_{1}}^{\psi}\left(h_{n}\right) \Delta_{\Psi}^{s_{2}} \sigma_{t_{1}+t_{2}}^{\psi}\left(h_{n}\right) \ldots \Delta_{\Psi}^{s_{m}} \sigma_{t_{1}+\cdots+t_{m}}^{\psi}\left(h_{n}\right) \Psi
$$

is holomorphic in $z=\left(s_{1}+i t_{1}, \ldots, s_{m}+i t_{m}\right)$ for $\operatorname{Re} z \in I_{m}^{1 / 2}$ and bounded uniformly for a bounded set of $h_{n}$, it has the equicontinuity in $\left(t_{1}, t_{1}+t_{2}\right.$, $\left.\ldots, t_{1}+\cdots+t_{m}\right)$ and hence

$$
\lim _{\beta \rightarrow+0} \Delta_{\Psi}^{s_{1}} h_{n}\left(f_{\beta}^{G}\right) \ldots \Delta_{\Psi}^{s_{m}} h_{n}\left(f_{\beta}^{G}\right) \Psi=\Delta_{\Psi}^{s_{1}} h_{n} \ldots \Delta_{\Psi}^{s_{m}} h_{n} \Psi
$$

strongly and uniformly over $n$ for each fixed $\left(s_{1} \ldots s_{m}\right)$ in $I_{m}^{1 / 2}$. The same equation holds when $h_{n}$ is replaced by $h$.

Furthermore,

$$
\Delta_{\Psi}^{s_{1}} h_{n}\left(f_{\beta}^{G}\right) \ldots \Delta_{\Psi}^{s_{m}} h_{n}\left(f_{\beta}^{G}\right) \Psi=h_{n}\left(f_{1}\right) \ldots h_{n}\left(f_{m}\right) \Psi
$$

where

$$
f_{j}(t)=f_{\beta}^{G}\left(t+i\left(s_{1}+\cdots+s_{j}\right)\right) .
$$

Hence by (4.3) we have 


$$
\begin{aligned}
\lim _{n \leftarrow 0} \Delta_{\Psi}^{s_{1}} h_{n}\left(f_{\beta}^{G}\right) \ldots \Delta_{\Psi}^{s_{m}} h_{n}\left(f_{\beta}^{G}\right) \Psi=h\left(f_{1}\right) \ldots h\left(f_{m}\right) \Psi \\
=\Delta_{\psi}^{s_{1}} h\left(f_{\beta}^{G}\right) \ldots \Delta_{\Psi}^{s_{m}} h\left(f_{\beta}^{G}\right) \Psi .
\end{aligned}
$$

Therefore, by taking the limit as $\beta \rightarrow+0$ and exchanging the order of limit in $n$ and, $\beta$, we obtain

$$
\lim _{n \rightarrow \infty} \Delta_{\Psi}^{s_{1}} h_{n} \ldots \Delta_{\Psi}^{s_{m}} h_{n} \Psi=\Delta_{\Psi}^{s_{1}} h \ldots \Delta_{\Psi}^{s_{m}} h \Psi
$$

for each $\left(s_{1} \ldots s_{m}\right) \in I_{m}^{1 / 2}$.

Since $\Delta_{\Psi}^{s_{1}} h_{n} \ldots \Delta_{\Psi}^{s m} h_{n} \Psi$ is uniformly bounded by $\left(\sup _{n}\left\|h_{n}\right\|\right)^{m}\|\Psi\|$, we have

$$
\lim _{n} \Psi\left(h_{n}\right)=\Psi(h)
$$

by Lebesgue dominated convergence theorem.

We now show that $\Psi(h) \in V_{\Psi}$. Let $h_{\beta}=h\left(f_{\beta}^{G}\right)$. Then $\left\|h_{\beta}\right\| \leqq\|h\|$ and $\lim _{\beta \rightarrow+0} h_{\beta}=h$. If we show $\Psi\left(h_{\beta}\right) \in V_{\Psi}$, then $\Psi(h)=\lim \Psi\left(h_{\beta}\right) \in V_{\Psi}$.

The closure of $\Delta_{\Psi}^{s} h_{\beta} \Delta_{T}^{-s}$ is given by

$$
h_{\beta}(-i s)=\int \Delta_{\Psi}^{i t} h \Delta_{\bar{T}}^{i t} f_{\beta}^{G}(t+i s) \mathrm{d} t \in M
$$

Hence

$$
\Delta_{\Psi}^{t_{n}} h_{\beta} \ldots \Delta_{\Psi}^{t_{1}} h_{\beta} \Psi=h_{\beta}\left(-i t_{n}\right) \ldots h_{\beta}\left(-i\left(t_{1}+\cdots+t_{n}\right)\right) \Psi
$$

Changing integration variables to $s_{1}=t_{1}+\cdots+t_{n}, s_{2}=t_{2}+\cdots+t_{n}, \ldots, s_{n}=t_{n}$, we obtain

$$
\begin{aligned}
\Psi\left(h_{\beta}\right) & =\sum_{n=0}^{\infty} \int_{0}^{1 / 2} \mathrm{~d} s_{1} \int_{0}^{s_{1}} \mathrm{~d} s_{2} \ldots \int_{0}^{s_{n-1}} \mathrm{~d} s_{n} h_{\beta}\left(-i s_{n}\right) \ldots h_{\beta}\left(-i s_{1}\right) \Psi \\
& =\operatorname{Exp}_{r}\left(\int_{0}^{1 / 2} ; h_{\beta}(-i s) \mathrm{d} s\right) \Psi
\end{aligned}
$$

where the expansional $\operatorname{Exp}_{r}$ is defined in [3].

By formula (3.5) of [3], we have

$$
\begin{aligned}
& \operatorname{Exp}_{r}\left(\int_{0}^{1 / 2} ; h_{\beta}(-i s) \mathrm{d} s\right) \\
& \quad=\operatorname{Exp}_{r}\left(\int_{0}^{1 / 4} ; h_{\beta}(-i s) \mathrm{d} s\right) \operatorname{Exp}_{r}\left(\int_{0}^{1 / 4} ; h_{\beta}(-i(s+1 / 4)) \mathrm{d} s\right)
\end{aligned}
$$


Since

$$
\begin{aligned}
h_{\beta}\left(-i\left(s_{n}+1 / 4\right)\right) \ldots h_{\beta}\left(-i\left(s_{1}+1 / 4\right)\right) \Psi \\
\quad=\Delta_{\Psi}^{1 / 2} h_{\beta}\left(-i\left(s_{n}-1 / 4\right)\right) \ldots h_{\beta}\left(-i\left(s_{1}-1 / 4\right)\right) \Psi \\
=J_{\Psi} h_{\beta}\left(i\left(s_{1}-1 / 4\right)\right) \ldots h_{\beta}\left(i\left(s_{n}-1 / 4\right)\right) \Psi,
\end{aligned}
$$

we have

$$
\operatorname{Exp}_{r}\left(\int_{0}^{1 / 4} ; h_{\beta}(-i(s+1 / 4)) \mathrm{d} s\right) \Psi=J_{\Psi} \operatorname{Exp}_{l}\left(\int_{0}^{1 / 4} ; h_{\beta}(i(s-1 / 4)) \mathrm{d} s\right) \Psi
$$

By formula (2.10) of [3], we have

$$
\operatorname{Exp}_{l}\left(\int_{0}^{1 / 4} ; h_{\beta}(i(s-1 / 4)) \mathrm{d} s\right)=\operatorname{Exp}_{r}\left(\int_{0}^{1 / 4} ; h_{\beta}(-i s) \mathrm{d} s\right) .
$$

Hence

$$
\Psi\left(h_{\beta}\right)=Q j_{\Psi}(Q) \Psi \in V_{\Psi}
$$

where

$$
Q=\operatorname{Exp}_{r}\left(\int_{0}^{1 / 4} ; h_{\beta}(-i s) \mathrm{d} s\right), j_{\Psi}(Q)=J_{\Psi} Q J_{\Psi}
$$

Q.E.D.

Definition 4.2. Let $\varphi \in \mathfrak{M}_{*}^{+}, \psi \in \mathfrak{M}_{*}^{+}$and $\xi_{\varphi}$, $\xi_{\psi}$ be unique representatives of $\varphi$ and $\phi$ in $V_{\Psi}$. If $\xi_{\varphi}=\xi_{\psi}(-h), h \in \mathfrak{M}, h=h^{*}$, then $h$ is called a Hamiltonian of $\varphi$ relative to $\phi$ and denoted by $\mathrm{h}(\varphi / \psi)$.

We shall prove the uniqueness in Lemma 4.6.

Remark. There is an unfortunate small discrepancy in the notation of mathematicians and physicists. As explained in Introduction, the time translation automorphism of physicists differs from the modular automorphisms of mathematicians by sign of the variable, which also causes a sign change in the statement of $K M S$ condition. In the present definition, it would be simpler mathematically to call $-h$ as a relative hamiltonian, but we would like to avoid a further discrepancy in terminologies and we define the hamiltonian as it appears in statistical mechanics. In the present 
article, we follow mathematician's notation regarding modular automorphisms, MSK conditions and inner product of a Hilbert space.

Proposition 4.3. Assume that $\mathrm{h}(\varphi / \psi)$ exists for faithful $\varphi$ and $\psi$ in $\mathfrak{M}_{*}^{+}$. Let $\sigma_{t}^{\varphi}$ and $\sigma_{t}^{\psi}$ be modular automorphisms for $\varphi$ and $\psi$. Let

$$
\begin{aligned}
& u_{t}^{\varphi \psi}=\operatorname{Exp}_{r}\left(\int_{0}^{t} ;-i \sigma_{s}^{\psi}(\mathrm{h}(\varphi / \psi)) \mathrm{d} s\right), \\
& \hat{u}_{t}^{\varphi \psi}=\operatorname{Exp}_{l}\left(\int_{0}^{t} ; i \sigma_{s}^{\psi}(\mathrm{h}(\varphi / \psi)) \mathrm{d} s\right) .
\end{aligned}
$$

Then

$$
\begin{aligned}
& \left(u_{t}^{\varphi \psi}\right)^{*}=\hat{u}_{t}^{\varphi \psi}, u_{t}^{\varphi \psi} \hat{u}_{t}^{\varphi \psi}=\hat{u}_{t}^{\varphi \psi} u_{t}^{\varphi \psi}=1 . \\
& u_{t}^{\varphi \psi} \sigma_{t}^{\psi}(x)=\sigma_{t}^{\varphi}(x) u_{t}^{\varphi \psi}, x \in \mathfrak{M} .
\end{aligned}
$$

Proof. The first equation in (4.8) follows from definition. The second equation in (4.8) follows from formulas (2.14) and (2.15) of [3]. To prove (4.9), we consider

$$
\varphi_{\beta}=\omega_{\xi_{\phi}\left(h_{\beta}\right)}
$$

where $h=-\mathrm{h}(\varphi / \psi), h_{\beta}=h\left(f_{\beta}^{G}\right)$ and $f_{\beta}^{G}$ is given by (3.11) of [2]. we first prove (4.9) for $\varphi_{\beta}$ instead of $\varphi$.

We have

$$
\begin{aligned}
& u_{t}^{\varphi{ } \psi}=\operatorname{Exp}_{r}\left(\int_{0}^{t} ; i h_{\beta}(s) \mathrm{d} s\right), \\
& \hat{u}_{t}^{\varphi{ } \psi}=\operatorname{Exp}_{l}\left(\int_{0}^{t} ;-i h_{\beta}(s) \mathrm{d} s\right),
\end{aligned}
$$

where

$$
h_{\beta}(z)=\int \sigma_{s}^{\Psi}(h) f_{\beta}^{G}(s-z) \mathrm{d} s \in \mathfrak{M} .
$$

We compare two functions

$$
\begin{aligned}
\mathrm{F}_{1}(t) & =\varphi_{\beta}\left(x u_{t}^{\varphi_{\beta} \psi} \sigma_{t}^{\psi}(y) \hat{u}_{t}^{\varphi_{\beta} \psi}\right) \\
& =\psi\left(A^{*} x A \sigma_{t}^{\psi}\left(k_{t}^{-1} y k_{t}\right)\right)
\end{aligned}
$$


and

$$
\begin{aligned}
\mathrm{F}_{2}(t) & =\varphi_{\beta}\left(u_{t}^{\varphi_{\beta} \psi} \sigma_{t}^{\psi t}(y) \hat{u}_{t}^{\varphi_{\beta} \psi} x\right) \\
& =\psi\left(\sigma_{t}^{\psi}\left(\hat{k}_{t}^{-1} y \hat{k}_{t}\right) A^{*} x A\right)
\end{aligned}
$$

where $x, y \in \mathfrak{M}$

$$
\begin{aligned}
& A=\operatorname{Exp}_{r}\left(\int_{0}^{1 / 2} ; h_{\beta}(-i s) \mathrm{d} s\right), \\
& k_{t}=\sigma^{\psi}{ }_{t}\left(\hat{u}_{t}^{\varphi^{\prime} \psi} A\right), \\
& \hat{k}_{t}=\sigma \underline{\psi}_{t}\left(\hat{u}_{t}^{\varphi^{\alpha} \psi}\left(A^{*}\right)^{-1}\right),
\end{aligned}
$$

and the second equalities of (4.11) and (4.12) are due to (4.5). We shall first prove that $k_{t}$ and $\hat{k}_{t}$ have analytic continuations to the same entire function and $k_{t-i}=\hat{k}_{t}$.

By formulas (4.2) and (4.4) of [3], we have

$$
\begin{aligned}
& \operatorname{Exp}_{r}\left(\int_{0}^{t+s} ;-i h_{\beta}(-\sigma) \mathrm{d} \sigma\right) \\
& \quad=\operatorname{Exp}_{r}\left(\int_{0}^{t} ;-i h_{\beta}(-\sigma) \mathrm{d} \sigma\right) \sigma^{\underline{\mu}} t\left\{\operatorname{Exp}_{r}\left(\int_{0}^{s} ;-i h_{\beta}(-\sigma) \mathrm{d} \sigma\right)\right\} .
\end{aligned}
$$

By formula (2.8) of $[3]$, we have

$$
\operatorname{Exp}_{r}\left(\int_{0}^{t} ;-i h_{\beta}(-\sigma) \mathrm{d} \sigma\right)=\operatorname{Exp}_{r}\left(\int_{0}^{1} ;-i t h_{\beta}(-t \sigma) \mathrm{d} \sigma\right)
$$

The right hand side is an entire function of $t$ and its value at $t= \pm i s$ is given by

$$
\operatorname{Exp}_{r}\left(\int_{0}^{1} ;-i t h_{\beta}(-t \sigma) \mathrm{d} \sigma\right)=\operatorname{Exp}_{r}\left(\int_{0}^{s} ; \pm h_{\beta}(\mp i \sigma) \mathrm{d} \sigma\right),
$$

due to formula (2.8) of [3] where $s$ is real positive. Hence the analytic continuation of $\operatorname{Exp}_{r}\left(\int_{0}^{t} ;-i h_{\beta}(-\sigma) \mathrm{d} \sigma\right)$ to $z=t \pm i s$ is given by

$$
\begin{aligned}
& \operatorname{Exp}_{r}\left(\int_{0}^{t} ;-i h_{\beta}(-\sigma) \mathrm{d} \sigma\right) \sigma{ }_{-t}\left\{\operatorname{Exp}_{r}\left(\int_{0}^{s} ; \pm h_{\beta}(\mp i \sigma) \mathrm{d} \sigma\right)\right\} \\
\equiv & \operatorname{Exp}_{r}\left(\int_{0}^{z} ;-i h_{\beta}(-\sigma) \mathrm{d} \sigma\right) .
\end{aligned}
$$


By formula (2.11) of [3], we have

$$
\begin{aligned}
\sigma_{\underline{\psi}_{t}}\left(\hat{u}_{t}^{\varphi_{\beta} \psi}\right) & =\operatorname{Exp}_{l}\left(\int_{0}^{t} ;-i h_{\beta}(\sigma-t) \mathrm{d} \sigma\right) \\
& =\operatorname{Exp}_{r}\left(\int_{0}^{t} ;-i h_{\beta}(-\sigma) \mathrm{d} \sigma\right) .
\end{aligned}
$$

Hence

$$
k_{t}=\operatorname{Exp}_{r}\left(\int_{0}^{t+i / 2} ;-i h_{\beta}(-\sigma) \mathrm{d} \sigma\right)
$$

We also have

$$
\begin{aligned}
\left(A^{*}\right)^{-1} & =\left(\operatorname{Exp}_{l}\left(\int_{0}^{1 / 2} ; h_{\beta}(i \sigma) \mathrm{d} \sigma\right)\right)^{-1} \\
& =\operatorname{Exp}_{r}\left(\int_{0}^{1 / 2} ;-h_{\beta}(i \sigma) \mathrm{d} \sigma\right)
\end{aligned}
$$

due to $h_{\beta}(-i \sigma)^{*}=h_{\beta}(i \sigma)$ and formulas (2.17), (2.14) and (2.15) of [3]. Hence

$$
\hat{k}_{t}=\operatorname{Exp}_{r}\left(\int_{0}^{t-i / 2} ;-i h_{\beta}(-\sigma) \mathrm{d} \sigma\right)
$$

Therefore $k_{t}$ and $\hat{k}_{t}$ have analytic continuations to the same entire function and $k_{t-i}=\hat{k}_{t}$.

$$
\text { Since } \begin{array}{ll}
\operatorname{Exp}_{r}\left(\int_{0}^{z} ;-i h_{\beta}(-\sigma) \mathrm{d} \sigma\right) \text { has an inverse } \\
& \left\{\sigma_{\underline{t}_{t}} \operatorname{Exp}_{l}\left(\int_{0}^{s} ; \mp h_{\beta}(\mp i \sigma) \mathrm{d} \sigma\right)\right\} \operatorname{Exp}_{l}\left(\int_{0}^{t} ; i h_{\beta}(-\sigma) \mathrm{d} \sigma\right)
\end{array}
$$

for all $z=t \pm i s, k_{t}^{-1}$ and $\hat{k}_{t}^{-1}$ have analytic continuations to the same entire function and $k_{t-i}^{-1}=\hat{k}_{t}^{-1}$.

Returning to $\mathrm{F}_{1}(t)$, it has an analytic continuation to $z=t-i / 2$ :

$$
\begin{aligned}
\mathrm{F}_{1}(t-i / 2) & =\left(\Delta_{\Psi}^{1 / 2} \sigma_{t}^{\psi}\left(k_{z}^{-1} y k_{z}\right) \Psi,\left(A^{*} x A\right) * \Psi\right) \\
& =\left(J_{\Psi} \sigma_{t}^{\psi}\left(k_{z}^{-1} y k_{z}\right) * \Psi,\left(A^{*} x A\right) * \Psi\right) \\
& =\left(J_{\Psi}\left(A^{*} x A\right)^{*} \Psi, \sigma_{t}^{\psi}\left(k_{z}^{-1} y k_{z}\right) * \Psi\right) .
\end{aligned}
$$


On the other hand $\mathrm{F}_{2}(t)$ has an analytic continuation to $\bar{z}=t+i / 2$ :

$$
\begin{aligned}
\mathrm{F}_{2}(t+i / 2) & =\left(A^{*} x A \Psi, \Delta_{\Psi}^{1 / 2} \sigma_{t}^{\psi}\left(\hat{k}_{\bar{z}}^{-1} y \hat{k}_{\bar{z}}\right) * \Psi\right) \\
& =\left(J_{\Psi}\left(A^{*} x A\right)^{* \Psi}, \sigma_{t}^{\psi}\left(\hat{k}_{\bar{z}}^{-1} y \hat{k}_{\bar{z}}\right) * \Psi\right) .
\end{aligned}
$$

Since $k_{z}=\hat{k}_{\bar{z}}$, they coincide. Hence the $*$-automorphism

$$
x \rightarrow u_{t}^{\varphi} \beta^{\psi} \sigma_{t}^{\psi}(x) \hat{u}_{t}^{\varphi^{\psi} \psi}
$$

satisfies the KMS condition for $\varphi_{\beta}$. Hence it must be the modular automorphism of $\varphi_{\beta}$ :

$$
u_{t}^{\varphi_{\beta}{ }^{\prime}} \sigma_{t}^{\psi}(x) \hat{u}_{t}^{\varphi^{\psi} \psi}=\sigma_{t}^{\varphi_{\beta}}(x)
$$

If $\beta \rightarrow+0, h_{\beta}$ tends to $?_{c}^{L}$ strongly. By Proposition 4.1, $\Psi\left(h_{\beta}\right)$ tends to $\Psi(h)$ strongly and hence $\varphi_{\beta}$ tends to $\varphi$ in norm. By Theorem 10 of $[2], \sigma_{t}^{\varphi_{\beta}}(x)$ tends to $\sigma_{t}^{\varphi}(x)$ strongly for $x \in \mathfrak{M}$.

On the other hand, $\sigma_{t_{1}}^{\prime}\left(h_{\beta}\right) \ldots \sigma_{t_{n}}^{\mathrm{\prime}}\left(h_{\beta}\right)$ converges strongly to $\sigma_{t_{1}}^{\mathrm{\prime}}(h) \ldots$ $\sigma_{t_{n}}^{\psi}(h)$ and hence $u_{t}^{\phi^{q} \psi}$ and $\hat{u}_{t}^{\phi_{\beta} \psi}$ converge strongly to $u_{t}^{\varphi \psi}$ and $\hat{u}_{t}^{\varphi \psi}$ by Lebesgue dominated convergence theorem. Hence we have

$$
u_{t}^{\varphi \psi} \sigma_{t}^{\psi}(x) \hat{u}_{t}^{\varphi \psi}=\sigma_{t}^{\varphi}(x), \quad x \in \mathfrak{M}
$$

which proves (4.9).

Q.E.D.

Corollary 4.4. If $\Psi$ is cyclic and separating and $h \in \mathfrak{M}, h^{*}=h$, then $\Psi(h)$ is also cyclic and separating.

Proof. By the proof of Proposition 4.3, $\Psi(h)$ satisfies KMS condition relative to the group of $*$-automorphisms

$$
x \in \mathfrak{M} \rightarrow u_{t} \sigma_{t}^{中}(x) u_{t}^{*}=\hat{\sigma}_{t}(x)
$$

where

$$
u_{t}=\operatorname{Exp}_{r}\left(\int_{0}^{t} ; i \sigma_{s}^{\psi}(h) \mathrm{d} s\right)
$$

and the group property of the *-automorphisms follow from the cocycle equation

$$
u_{t_{1}} \sigma_{t_{1}}\left(u_{t_{2}}\right)=u_{t_{1}+t_{2}}
$$


which is formula (4.2) of [3]. It is known that a KMS state is faithful if it is faithful on the center.

Let $E$ be a central projection. Then $E\{\Psi(h)\}=(E \Psi)(E h)$, where we restrict our attention to $E M$ and the restriction $E \Delta_{\Psi}$ of $\Delta_{\Psi}$ (which commutes with $E$ ) is $\Delta_{E \Psi}$. Since $\Psi$ is separating $E \Psi \neq 0$ unless $E=0$.

We now prove $\Phi(h) \neq 0$ for any non-zero cyclic and separating $\Phi$. We then have $E \Psi(h) \neq 0$ and hence $\Psi(h)$ is separating for $M$. Since $\Psi(h) \in$ $V_{\Psi}, \Psi(h)$ is then also cyclic and the proof is complete.

To prove $\Phi(h) \neq 0$, we define

$$
\Phi_{z}(h) \equiv \sum_{n=0}^{\infty} z^{n} \int_{0}^{1} \mathrm{~d} s_{1} \ldots \int_{0}^{s_{n-1}} \mathrm{~d} s_{n} \Delta_{\Phi}^{s_{n} z} h \Delta_{\Phi}^{\left(s_{n-1}-s_{n}\right) z} \ldots \Delta_{\Phi}^{\left(s_{1}-s_{2}\right) z} h \Phi .
$$

The integrand is continuous for $\operatorname{Re} z \in[0,1 / 2]$, holomorphic for $\operatorname{Re} z \in(0$, $1 / 2$ ) and uniformly bounded by $\|h\|^{n}\|\Phi\|$ for $\operatorname{Re} z \in[0,1 / 2]$. Hence $\Phi_{z}(h)$ is continuous for $\operatorname{Re} z \in[0,1 / 2]$ and holomorphic for $\operatorname{Im} z \in(0,1 / 2)$.

Next we prove the following formula:

$$
\Phi_{z+i t}(h)=u_{t} \Delta_{\emptyset}^{i t} \Phi_{z}(h)
$$

where $t$ is real,

$$
u_{t}=\operatorname{Exp}_{r}\left(\int_{0}^{t} ; i \sigma_{s}^{\varphi}(h) \mathrm{d} s\right)
$$

and $\varphi=\omega_{0}$. If this formula is proved, then $\Phi(h)=\Phi_{1 / 2}(h)=0$ implies $\Phi_{1 / 2+i t}(h)=0$ and hence $\Phi_{z}(h)=0$ by the edge of wedge theorem. In particular $\Phi_{0}(h)=\Phi=0$. Since $\Phi \neq 0$ by assumption, we obtain $\Phi(h) \neq 0$.

Since $u_{t}$ is a unitary operator strongly continuous in $h$ and $\Phi_{z}(h)$ is also strongly continuous in $h$ by the proof of Proposition 4.1, it is enough to prove the formula when $h$ is replaced by $h_{\beta}=h\left(f_{\beta}^{G}\right)$. For $h_{\beta}$, the formula reduces to

$$
\begin{aligned}
& \operatorname{Exp}_{r}\left(\int_{0}^{1} ;(z+i t) \sigma_{-i(z+i t) s}\left(h_{\beta}\right) \mathrm{d} s\right) \\
& =\operatorname{Exp}_{r}\left(\int_{0}^{t} ; i \sigma_{s}^{\varphi}\left(h_{\beta}\right) \mathrm{d} s\right) \sigma_{t}^{\varphi}\left\{\operatorname{Exp}_{r}\left(\int_{0}^{1} ; z \sigma_{-i z s}^{\varphi}(h) \mathrm{d} s\right)\right\},
\end{aligned}
$$

which holds for $\operatorname{Re} z=0$ due to the formula (2.8) and (4.2) of [3] and hence for $\operatorname{Re} z \in[0,1 / 2]$ by the edge of wedge theorem.

Q.E.D. 
Proposition 4.5. If $\Phi=\Psi(h)$, then $\Phi(-h)=\Psi$. If $\Phi_{1}=\Psi\left(h_{1}\right)$ and $\Phi_{2}=\Phi_{1}\left(h_{2}\right)$, then $\Phi_{2}=\Psi\left(h_{1}+h_{2}\right)$.

Proof. First we consider

$$
\begin{aligned}
h_{1 \beta} & =\int \Delta_{\dot{\Psi}}^{i t} h_{1} \Delta_{\bar{\Psi}}^{-i t} f_{\beta}^{G}(t) \mathrm{d} t, \Phi_{1 \beta}=\Psi\left(h_{1 \beta}\right), \\
h_{2 \gamma \beta} & =\int \Delta_{\Phi_{1 \beta}}^{i t} h_{2} \Delta_{\Phi_{1 \beta}}^{-i t} f_{\gamma}^{G}(t) \mathrm{d} t, \Phi_{2 \beta \gamma}=\Phi_{1 \beta}\left(h_{2 \gamma \beta}\right) .
\end{aligned}
$$

Then we have

$$
\begin{aligned}
& \Phi_{1 \beta}=\operatorname{Exp}_{r}\left(\int_{0}^{1 / 2} ; h_{1 \beta}(-i s) \mathrm{d} s\right) \Psi \\
& \Phi_{2 \beta \gamma}=\operatorname{Exp}_{r}\left(\int_{0}^{1 / 2} ; h_{2 \gamma \beta}(-i s) \mathrm{d} s\right) \Phi_{1 \beta}
\end{aligned}
$$

where $h_{1 \beta}(z)$ and $h_{2 \gamma \beta}(z)$ are analytic continuations of $h_{1 \beta}(t)=\sigma_{t}^{\prime \prime}\left(h_{1 \beta}\right)$ and $h_{2 \gamma \beta}(t)=\sigma_{t}^{\varphi_{1 \beta}}\left(h_{2 \gamma \beta}\right)$, and $\psi=\omega_{\Psi}, \varphi_{1 \beta}=\omega_{\mathscr{\omega}_{1 \beta}}$.

By Proposition 4.3, we have

$$
\begin{aligned}
& \sigma_{t}^{t^{\prime}}(x)=u_{t}^{-1} \sigma_{t}^{\phi_{1 \beta}}(x) u_{t} \\
& u_{t}=\operatorname{Exp}_{r}\left(\int_{0}^{t} ; i h_{1 \beta}(s) \mathrm{d} s\right)=\operatorname{Exp}_{r}\left(\int_{0}^{1} ; i t h_{1 \beta}(t s) \mathrm{d} s\right) .
\end{aligned}
$$

By analytic continuation of the right hand side, we obtain the analytic continuation of the left hand side:

$$
\sigma_{z}^{\psi}\left(h_{2 \gamma \beta}\right)=\operatorname{Exp}_{r}\left(\int_{0}^{1} ; i z h_{1 \beta}(z s) \mathrm{d} s\right)^{-1} h_{2 \gamma \beta}(z) \operatorname{Exp}_{r}\left(\int_{0}^{1} ; i z h_{1 \beta}(z s) \mathrm{d} s\right)
$$

Hence

$$
h_{2 \gamma \beta}(-i t)=\operatorname{Exp}_{r}\left(\int_{0}^{t} ; h_{1 \beta}(-i s) \mathrm{d} s\right) \sigma \underline{\psi}_{i t}\left(h_{2 \gamma \beta}\right) \operatorname{Exp}_{r}\left(\int_{0}^{t} ; h_{1 \beta}(-i s) \mathrm{d} s\right)^{-1}
$$

By formula (3.10) of [3],

$$
\begin{aligned}
\Phi_{2 \gamma \beta} & =\operatorname{Exp}_{r}\left(\int_{0}^{1 / 2} ;\left\{h_{1 \beta}(-i s)+\sigma \underline{w}_{i s}\left(h_{2 \gamma \beta}\right)\right\} \mathrm{d} s\right) \Psi . \\
& =\Psi\left(h_{1 \beta}+h_{2 \gamma \beta}^{\prime}\right) .
\end{aligned}
$$


In the limit $\beta \rightarrow+0, h_{1 \beta}$ tends to $h_{1}$, and hence $\Phi_{1 \beta}$ tends to $\Phi_{1}$ strongly. By the proof of Theorem 10 of $[2], \Delta_{\omega_{1 \beta}}^{i t}$ tends to $\Delta_{\omega_{1}}^{i t}$ strongly (uniformly over bounded $t$ ) and hence

$$
h_{2 \gamma \beta}(z)=\int \Delta_{\tilde{\Phi}_{1 \beta}}^{i t} h_{2} \Delta_{\tilde{\Phi}_{1 \beta}}^{-i t} f_{\gamma}^{G}(t-z) \mathrm{d} t
$$

tends to

$$
h_{2 \gamma}(z)=\int \Delta_{\tilde{\omega}_{1}}^{i t} h_{2} \Delta_{\tilde{\omega}_{1}}^{i t} f_{\gamma}^{G}(t-z) \mathrm{d} t
$$

which is an analytic continuation of

$$
\sigma_{t}^{\varphi_{1}}\left(h_{2 \gamma}\right), h_{2 \gamma}=\int \Delta_{\Phi_{1}}^{i t} h_{2} \Delta_{\tilde{\omega}_{1}}^{-i t} f_{\gamma}^{G}(t) \mathrm{d} t
$$

Therefore $h_{2 \gamma \beta}$ tends to $h_{2 \gamma}$ strongly and $\Phi_{1 \beta}\left(h_{2 \gamma \beta}\right)$ tends strongly to $\Phi_{1}\left(h_{2 \gamma}\right)$. By Proposition 4.1, $\Psi\left(h_{1 \beta}+h_{2 \gamma \beta}\right)$ tends to $\Psi\left(h_{1}+h_{2 \gamma}\right)$. Hence

$$
\Phi_{1}\left(h_{2 \gamma}\right)=\Psi\left(h_{1}+h_{2 \gamma}\right) \text {. }
$$

In the limit $\gamma \rightarrow+0, h_{2 \gamma}$ tends to $h_{2}$ and hence by Proposition 4.1

$$
\Phi_{1}\left(h_{2}\right)=\Psi\left(h_{1}+h_{2}\right)
$$

By taking $h_{2}=-h_{1}$, we have $\Phi(-h)=\Psi$ when $\Phi=\Psi(h)$.

Proposition 4.6. For given faithful $\psi$ and $\varphi \in \mathfrak{M}_{*}^{+}, \mathrm{h}(\varphi / \psi)$ is unique if it exists.

Proof. Any cone $V_{\Psi}$ is related to any other cone $V_{\Psi^{\prime}}$ by a unitary $u^{\prime} \in \mathfrak{M l}^{\prime}: u^{\prime} V_{\Psi}=V_{\Psi^{\prime}}$. Hence the choice of $V_{\Psi}$ does not affect the definition of $\mathrm{h}(\varphi / \psi)$. We fix one $V_{\Psi}$. Assume that

$$
\Psi\left(h_{1}\right)=\Phi=\Psi\left(h_{2}\right), \omega_{\Psi}=\psi, \omega_{\Phi}=\varphi \text {. }
$$

By Proposition 4.5,

$$
\Psi\left(h_{1}-h_{2}\right)=\Phi\left(-h_{2}\right)=\Psi
$$

By Proposition 4.3, $\operatorname{Exp}_{r}\left(\int_{0}^{t} ; i \sigma_{s}^{\psi}\left(h_{1}-h_{2}\right) \mathrm{d} s\right)$ must commute with all $\sigma_{t}^{\psi}(x)$, 
$x \in \mathfrak{M}$ and hence is in the center of $\mathfrak{M}$. By differentiating by $t$ at $t=0$, we see that $h_{1}-h_{2}$ is in the center of $\mathfrak{M}$. We then have $\sigma_{t}^{\text {t) }}\left(h_{1}-h_{2}\right)=$ $h_{1}-h_{2}$ and hence

$$
\Psi\left(h_{1}-h_{2}\right)=\exp \left\{\left(h_{1}-h_{2}\right) / 2\right\} \Psi=\Psi
$$

Since $\Psi$ is separating, we have $h_{1}-h_{2}=0$ for selfadjoint $h_{1}-h_{2}$.

Q.E.D.

Proposition 4.7. $\hat{u}_{t}^{\varphi \psi t}=u_{t}^{\varphi \psi}$.

Proof. By (4.9), we have

$$
\begin{aligned}
& u_{t}^{\varphi \varphi}=\operatorname{Exp}_{r}\left(\int_{0}^{t} ; u_{s}^{\varphi \downarrow}\left(-i \sigma_{s}^{\uparrow}(\mathrm{h}(\psi / \varphi))\right)\left(u_{s}^{\varphi \psi}\right)^{-1} \mathrm{~d} s\right) \\
& u_{t}^{\varphi t}=\operatorname{Exp}_{r}\left(\int_{0}^{t} ;-i \sigma_{s}^{\uparrow}(\mathrm{h}(\varphi / \psi)) \mathrm{d} s\right) .
\end{aligned}
$$

By formula (3.10) of [3], we have

$$
u_{t}^{\varphi \phi} u_{t}^{\varphi \phi}=\operatorname{Exp}_{r}\left(\int_{0}^{t} ;-i \sigma_{s}^{\phi}(h(\psi / \varphi)+h(\varphi / \psi)) \mathrm{d} s\right) .
$$

By Proposition 4.5, the right hand side is 1 and hence

$$
u_{t}^{\mid \psi \varphi}=u_{t}^{\psi \mid \varphi} u_{t}^{\varphi \psi \mid} \hat{u}_{t}^{\varphi \psi t}=\hat{u}_{t}^{\varphi \psi} .
$$

Q.E.D.

Proposition 4.8. Let $\Phi=\Psi(-h), h \in \mathfrak{M}, h^{*}=h, H_{\Psi}=-\log \Delta_{\Psi}, H_{\varpi}=$ $-\log \Delta_{\emptyset}$. Then

$$
H_{\varpi}=H_{\Psi}+h-j(h)
$$

where $j(h)=J_{\Psi} h J_{\Psi}=J_{\Phi} h J_{\emptyset} . \quad$ For $\omega_{\emptyset}=\varphi, \omega_{\Psi}=\psi$,

$$
\begin{aligned}
& u_{t}^{\varphi \psi} \Delta_{\Psi}^{i t}=\exp \left(-i t\left(H_{\Psi}+h\right)\right)=j\left(u_{t}^{\dagger \cdot \varphi}\right) \Delta_{\oplus}^{i t}, \\
& \Delta_{\bar{\Phi}}^{-i t} u_{t}^{\varphi \uparrow}=\exp \left(i t\left(H_{\varpi}-h\right)\right)=\Delta_{\bar{\Psi}}^{i t} j\left(u_{t}^{\dagger \cdot \varphi}\right), \\
& \Delta_{\oplus}^{i t}=j\left(u_{t}^{\varphi t)}\right) u_{t}^{\varphi \downarrow} \Delta_{\Psi}^{i t}=\Delta_{\Psi}^{i t} j\left(u_{-t}^{\dagger \cdot \varphi}\right) u_{-t}^{\phi \varphi} .
\end{aligned}
$$


Proof. By Remark to Proposition 16 of [3] and equation (4.6), we have the first equality of (4.14) where $H_{\Psi}+h$ is selfadjoint. By Proposition 4.7 and equation (4.8), we have

$$
u_{t}^{\varphi \psi}=\left(u_{t}^{\varphi \varphi}\right)^{*}=\operatorname{Exp}_{l}\left(\int_{0}^{t} ;-i \sigma_{s}^{\varphi}(h) \mathrm{d} s\right)
$$

Hence by Remark to Proposition 16 of [3], we have the first equality of (4.15), where $H_{\varpi}-h$ is selfadjoint. ((4.15) can be obtained also from (4.14) by taking adjoint and interchanging $\varphi$ and $\psi$.)

Consider

$$
w=\Delta_{\bar{\emptyset}}^{-i t} j\left(u_{t}^{\varphi \psi}\right) u_{t}^{\varphi \psi} \Delta_{\Psi}^{i t}
$$

For $x \in \mathfrak{M}$ and $y \in \mathfrak{M}^{\prime}$, we have

$$
\begin{aligned}
w x y & =w x j(j(y))=\Delta_{\tilde{\omega}}^{-i t} j\left(u_{t}^{\varphi t}\right) u_{t}^{\varphi \psi} \sigma_{t}^{\psi}(x) j\left(\sigma_{t}^{\psi}\{j(y)\}\right) \Delta_{\Psi}^{i t} \\
& =\Delta_{\tilde{\Phi}}^{-i t} \sigma_{t}^{\varphi}(x) j\left(\sigma_{t}^{\varphi}\{j(y)\}\right) j\left(u_{t}^{\varphi \psi}\right) u_{t}^{\varphi \psi t} \Delta_{\Psi}^{i t} \\
& =x j(j(y)) w=x y w .
\end{aligned}
$$

Hence $w \in \mathfrak{M} \cap \mathfrak{M}^{\prime}\left(=\left(\mathfrak{M} \cup \mathfrak{M}^{\prime}\right)^{\prime}\right)$. Obviously $w$ is unitary. Since $V_{\Psi}=$ $V_{\mathscr{\sigma}}$ is invariant under multiplication of $\Delta_{\Psi}^{i t}, \Delta_{\phi}^{i t}$ and $Q j(Q), Q=u_{t}^{\varphi \psi} \in \mathfrak{M}$, we have $w V_{\Psi} \subset V_{\Psi}$. By the next Lemma, this implies $w \geqq 0$ and hence $w=1$. Hence we have the first equality of (4.16). By taking adjoint and changing the sign of $t$, we obtain the second equality of (4.16). From $w=1$, we also obtain second equalities of (4.14) and (4.15).

By (4.14), (4.15) and $w=1$, we have

$$
\begin{aligned}
\exp \left(-i t\left(H_{\Psi}+h\right)\right) & =j\left(u_{t}^{\Phi \phi}\right) * \Delta_{\Phi}^{i t}=\left\{j\left(\Delta_{\Phi}^{-i t} u_{t}^{\varphi \phi}\right)\right\}^{*} \\
& =\exp \left(-i t\left(H_{\varpi}+j(h)\right)\right)
\end{aligned}
$$

where we have used the property $j\left(H_{\varpi}\right)=-H_{\varpi}$, which follows from $J_{\emptyset} \Delta_{\varpi} J_{\emptyset}$ $=\Delta_{\tilde{\Phi}}^{-1}$. Since both $H_{\Psi}+h$ and $H_{\Phi}+j(h)$ are selfadjoint operators, we have

$$
H_{\Psi}+h=H_{\emptyset}+j(h) \text {. }
$$

Since $H_{\Psi}+h$ and $H_{\Psi}+h-j(h)$ have the same domain, which also coincides with the domain of $H_{\emptyset}$ by (4.17), we have (4.13). Q.E.D. 
Lemma 4.9. If $w \in \mathfrak{M} \cap \mathfrak{M}^{\prime}$ and $w V_{\Psi} \subset V_{\Psi}$, then $w \geqq 0$.

Proof. Since $\mathfrak{M} \cap \mathfrak{M}^{\prime}$ is commutative, $w$ is normal. Let $E$ be a spectral projection of $w$ for an open set contained in the upper half complex plane. Since $E \in \mathfrak{M} \cap \mathfrak{M}^{\prime}, E j_{\Psi}(E)=E E^{*}=E$ by Lemma 3 of [2] and hence $E V_{\Psi} \subset V_{\Psi}$. Therefore, for any $\Phi \in V_{\Psi}$, we have $E \Phi \in V_{\Psi}, w \Phi \in V_{\Psi}$ and hence $\omega_{\mathscr{\omega}}(E w) \geqq 0$. On the other hand, $E$ is a spectral projection of $w$ for an open set in the upper half plane and hence $\operatorname{Im} \omega_{\varpi}(E w)>0$ unless $E \Phi=0$. Therefore we have $E \Phi=0$ for any $\Phi \in V_{\Psi}$ and hence $E=0$. Similarly $w$ can not have a spectrum in the lower half plane nor in the negative real axis. Hence $w \geqq 0$.

Proposition 4.10. If $\Phi \leqq l^{1 / 2} \Psi$ (i.e. $l^{1 / 2} \Psi-\Phi \in V_{\Psi}$ ) and if $\mathrm{h}(\varphi / \psi)$ exists for $\omega_{\emptyset}=\varphi, \omega_{\Psi}=\psi$ then $-\mathrm{h}(\varphi / \psi) \leqq \log l$.

Proof. For $h \in \mathfrak{M}, h^{*}=h$, we define

$$
\begin{array}{r}
\Psi(t ; h)=\sum_{n=0}^{\infty} \int_{0}^{t} \mathrm{~d} t_{1} \int_{0}^{t_{1}} \mathrm{~d} t_{2} \ldots \int_{0}^{t_{n-1}} \mathrm{~d} t_{n} \Delta_{\Psi}^{t_{n}} h \Delta_{\Psi}^{t_{n-1}-t_{n}} \ldots \Delta_{\Psi}^{t_{1}-t_{2}} h \Psi \\
0 \leqq t \leqq 1 / 2 .
\end{array}
$$

$\Psi(h)$ defined earlier is $\Psi(1 / 2 ; h)$. We shall first prove the following formula:

$$
\begin{aligned}
\omega^{t}(x) & \equiv\left(\Delta_{\Psi}^{(1-t) / 2} x \Psi, \Psi(t ; h)\right) \\
& =\left(x J_{\Psi} \Psi(t / 2 ; h), J_{\Psi} \Psi(t / 2 ; h)\right), x \in \mathfrak{M} .
\end{aligned}
$$

First consider $h_{\beta}=h\left(f_{\beta}^{G}\right)$ instead of $h$. Then

$$
\Psi\left(t ; h_{\beta}\right)=\operatorname{Exp}_{r}\left(\int_{0}^{t} ; h_{\beta}(-i s) \mathrm{d} s\right) \Psi .
$$

Hence

$$
\begin{aligned}
& \left(x J_{\Psi} \Psi\left(t / 2 ; h_{\beta}\right), J_{\Psi} \Psi\left(t / 2 ; h_{\beta}\right)\right)=\left(x \Psi, j_{\Psi}\left(k^{*}\right) j_{\Psi}(k) \Psi\right) \\
= & \left(x \Psi, j_{\Psi}\left(k^{*} k\right) \Psi\right)=\left(x \Psi, \Delta_{\Psi}^{1 / 2} k^{*} k \Psi\right) \\
= & \left(\Delta_{\Psi}^{(1-t) / 2} x \Psi, \Delta_{\Psi}^{t / 2} k^{*} k \Psi\right)
\end{aligned}
$$


where

$$
k=\operatorname{Exp}_{r}\left(\int_{0}^{t / 2} ; h_{\beta}(-i s) \mathrm{d} s\right)
$$

Since the closure of $\Delta_{\Psi}^{t / 2} k^{*} \Delta_{\Psi}^{t / 2}$ is given by

$$
\operatorname{Epx}_{l}\left(\int_{0}^{t / 2} ; h_{\beta}(i(s-t / 2)) \mathrm{d} s\right)=\operatorname{Exp}_{r}\left(\int_{0}^{t / 2} ; h_{\beta}(-i s) \mathrm{d} s\right)=k,
$$

due to formula (2.11) of [3], we obtain as the closure of $\Delta_{\Psi}^{t / 2} k^{*} k \Delta_{\bar{T}}^{-t / 2}$

$$
\begin{aligned}
& \operatorname{Exp}_{r}\left(\int_{0}^{t / 2} ; h_{\beta}(-i s) \mathrm{d} s\right) \operatorname{Exp}_{r}\left(\int_{0}^{t / 2} ; h_{\beta}(-i(t / 2+s)) \mathrm{d} s\right) \\
& \quad=\operatorname{Exp}_{r}\left(\int_{0}^{t} ; h_{\beta}(-i s) \mathrm{d} s\right)
\end{aligned}
$$

by formula (3.5) of [3]. Hence (4.19) is proved for $h=h_{\beta}$.

By taking the limit $\beta \rightarrow+0$, we obtain (4.19) by continuity of $\Psi(t ; h)$ on $h$, which can be proved in exctly the same way as the continuity of $\Psi(h)$ in $h$.

Assume now $\Phi=\Psi(h), \Phi \leqq l^{1 / 2} \Psi \quad$ For a general $\alpha \in[0,1 / 2]$, the closure of $\Delta_{\Psi}^{\alpha} \mathfrak{M} \mathcal{R}^{+} \Psi$ is denoted by $V_{\Psi}^{\alpha}$ in [2]. By Theorem 3 (5) of [2], it is dual to $V_{\Psi}^{1 / 2-\alpha}$. By Taking $x \in \mathfrak{M}^{+}$in (4.19), we obtain

$$
\Psi(t ; h) \in\left(V_{\Psi}^{1 / 2-t / 2}\right)^{\prime}=V_{\Psi}^{t / 2}
$$

We now prove

$$
l^{2^{-n}} \Psi-\Psi\left(2^{-n} ; h\right) \in V_{\Psi}^{2^{-(n+1)}}
$$

by induction on $n$. It is true for $n=1$ by our assumption.

Assume that

$$
l^{t} \Psi-\Psi(t ; h) \in V_{\Psi}^{t / 2}, \quad t \leqq 1 / 2
$$

Since $\Delta_{\Psi}^{(1-t) / 2} x \Psi \in V_{\Psi}^{1 / 2-t / 2}=\left(V_{\Psi}^{t / 2}\right)^{\prime}$ for $x \in \mathfrak{M}^{+}$, we have

$$
\omega^{t}(x) \leqq l^{t}\left(\Delta_{\Psi}^{(1-t) / 2} x \Psi, \Psi\right)=l^{t} \psi(x), x \in \mathfrak{M}^{+} .
$$

Hence $\omega_{\chi} \leqq l^{t} \psi$ for $\chi=J_{\Psi} \Psi(t / 2 ; h)$. Hence, there exists $y_{t} \in \mathfrak{M}^{\prime}$ such that

$$
J_{\Psi} \Psi(t / 2 ; h)=y_{t} \Psi,\left\|y_{t}\right\| \leqq l^{t / 2}
$$


Let $x_{t}=J_{\Psi} y_{t} J_{\Psi} \in \mathfrak{M}$.

By Theorem $3(2)$ of $[2]$ and equation (4.20), we have

$$
J_{\Psi} \Psi(t / 2 ; h)=\Delta_{\Psi}^{(1-t) / 2} \Psi(t / 2 ; h) .
$$

Hence

$$
\Delta_{\Psi}^{1 / 2} x_{t}^{*} \Psi=J_{\Psi} x_{t} \Psi=J_{\Psi} \Psi(t / 2 ; h)=\Delta_{\Psi}^{(1-t) / 2} x_{t} \Psi
$$

By Lemma 6 of $[2], \sigma_{s}^{\psi}\left(x_{t}\right)$ has an analytic continuation to $\operatorname{Im} s \in[0, t / 2)$ and $\left\|\sigma_{s}^{t}\left(x_{t}\right)\right\|_{1} \leqq\left\|x_{t}\right\| \leqq l^{t / 2}$ for the analytic continuation. In particular, $0 \leqq \sigma_{i t / 4}^{\prime \prime}\left(x_{t}\right) \leqq l^{t / 2}$ where the positivity comes from $\Psi(t / 2 ; h) \in V_{\Psi}^{t / 4}$ and Theorem $3(7)$ of $[2]$. Hence

$$
l^{t / 2} \Psi-\Psi(t / 2 ; h)=\Delta_{\Psi}^{t / 4}\left(l^{t / 2}-\sigma_{i t / 4}^{\psi}\left(x_{t}\right)\right) \Psi \in V_{\Psi}^{t / 4} .
$$

This completes the inductive proof of (4.21).

We have

$$
\begin{aligned}
& \lim _{n \rightarrow \infty}\left(l^{2^{-n}}-1\right) 2^{n}=\log l, \\
& \lim _{n \rightarrow \infty}\left\{\Psi\left(2^{-n} ; h\right)-\Psi\right\} 2^{n}=h \Psi,
\end{aligned}
$$

where the last equation is due to the estimate

$$
\begin{array}{r}
\left\|\int_{0}^{t} \mathrm{~d} t_{1} \ldots \int_{0}^{t_{n-1}} \mathrm{~d} t_{n} \Delta^{t_{n}} h \Delta^{t_{n-1}-t_{n}} \ldots \Delta^{t_{1}-t_{2}} h \Psi\right\| \\
\leqq(n !)^{-1} t^{n}\|h\|^{n}\|\Psi\|, \quad 0 \leqq t \leqq 1 / 2
\end{array}
$$

and

$$
\lim _{t \rightarrow 0} t^{-1} \int_{0}^{t} \mathrm{~d} s \Delta^{s} h \Psi=h \Psi
$$

Hence we have

$$
(\log l-h) \Psi=\lim _{n \rightarrow \infty} 2^{n}\left(l^{2^{-n}} \Psi-\Psi\left(2^{-n} ; h\right)\right)
$$

Since $\left\|\Delta^{1 / 2-t} x \Psi\right\| \leqq 2\|x\|\|\Psi\|$ for $x \in \mathfrak{M} \quad t \in[0,1 / 2]$, and $\Delta^{1 / 2-t} x \Psi$ is strongly continuous in $t \in[0,1 / 2]$, we have 


$$
\left(\Delta^{1 / 2} x \Psi, \Phi\right)=\lim _{n}\left({\Delta^{2-1}-2^{-(n+1)}}^{2} \Psi, \Phi_{n}\right)
$$

whenever $\left\|\Phi_{n}-\Phi\right\| \rightarrow 0$. By (4.22) and (4.21), we have

$$
\left(\Delta^{1 / 2} x \Psi,(\log l-h) \Psi\right) \geqq 0
$$

for all $x \in \mathfrak{M}^{+}$. Hence $(\log l-h) \Psi \in V_{\Psi}^{0}$ and we have

$\log l \geqq h$.

Q.E.D.

Corollary 4.11. If $l_{1}^{1 / 2} \Psi \leqq \Phi \leqq l_{2}^{1 / 2} \Psi, \varphi=\omega_{\emptyset}, \quad \psi=\omega_{\Psi}$, and $\mathrm{h}(\varphi / \psi)$ exists, then

$$
\log l_{1} \leqq-\mathrm{h}(\varphi / \psi) \leqq \log l_{2}
$$

Proof. By Proposition 4.10, we have $-\mathrm{h}(\varphi / \psi) \leqq \log l_{2}$. Since $\Psi \leqq$ $l_{1}^{-1 / 2} \Phi$, we have $-\mathrm{h}(\psi / \varphi) \leqq-\log l_{1}$. Since $\mathrm{h}(\varphi / \psi)=-\mathrm{h}(\psi / \varphi)$, we have $\log l_{1} \leqq-\mathrm{h}(\varphi / \psi)$.

Q.E.D.

Proposition 4.12. For $h \in \mathfrak{M}, h^{*}=h$, a cyclic and separating vector $\Psi$ is in the domain of $\exp z\left(-H_{\Psi}+h\right)$ for $\operatorname{Re} z \in[0,1 / 2]$, where $H_{\Psi}=$ $-\log \Delta_{\Psi}$, the vector

$$
\begin{aligned}
\Psi(z) \equiv & \exp z\left(-H_{\Psi}+h\right) \Psi=\sum_{n=0}^{\infty} z^{n} \int_{0}^{1} \mathrm{~d} s_{1} \int_{0}^{s_{1}} \mathrm{~d} s_{2} \ldots \int_{0}^{s_{n-1}} \mathrm{~d} s_{n} \\
& \Delta_{\Psi}^{s n z} h \Delta_{\Psi}^{\left(s_{n-1}-s_{n}\right) z} \ldots \Delta_{\Psi}^{\left(s_{1}-s_{2}\right) z} h \Psi
\end{aligned}
$$

is holomorphic in $z$ for $\operatorname{Re} z \in(0,1 / 2)$ and strongly continuous in $z$ for Im $z \in[0,1 / 2]$. If $h_{n}$ tends to $h$ strongly then

$$
\lim _{n} \exp z\left(-H_{\Psi}+h_{n}\right) \Psi=\exp z\left(-H_{\Psi}+h\right) \Psi
$$

strongly for $\operatorname{Re} z \in[0,1 / 2]$.

Proof. For $z=i t$ with real $t$, we have

$$
\sum_{n=0}^{\infty} z^{n} \int_{0}^{1} \mathrm{~d} s_{1} \int_{0}^{s_{1}} \mathrm{~d} s_{2} \ldots \int_{0}^{s_{n-1}} \mathrm{~d} s_{n} \Delta_{\Psi}^{s n z} h \Delta_{\Psi}^{\left(s_{n-1}-s_{n}\right) z} \ldots \Delta_{\Psi}^{\left(s_{1}-s_{2}\right) z} h \Psi
$$




$$
\begin{aligned}
& =\sum_{n=0}^{\infty} i^{n} \int_{0}^{t} \mathrm{~d} t_{1} \int_{0}^{t_{1}} \mathrm{~d} t_{2} \ldots \int_{0}^{t_{n-1}} \mathrm{~d} t_{n} \sigma_{t_{n}}^{\psi}(h) \ldots \sigma_{t_{1}}^{\psi}(h) \Psi \\
& =u_{t}^{\varphi \psi} \Psi=u_{t}^{\varphi \psi} \Delta_{\Psi}^{i t} \Psi=\exp i t\left(-H_{\Psi}+h\right) \Psi
\end{aligned}
$$

where $\Phi=\Psi(h), \varphi=\omega_{\varpi}, \psi=\omega_{\Psi}$ and Proposition 4.8 is used. Hence (4.24) holds for pure imaginary $z$.

If $H$ is any selfadjoint operator and $e^{z H \Psi}$ with pure imaginary $z$ has an "analytic continuation" $\Psi(z)$ holomorphic for $\operatorname{Re} z \in(0, \delta)$ and continuous for $\operatorname{Re} z \in[0, \delta]$, then $\Psi$ is in the domain of $e^{z H}, \operatorname{Re} z \in[0, \delta]$ and $\Psi(z)=e^{z H} \Psi$ due to the following argument:

Let $H=\int \lambda \mathrm{d} E_{\lambda}$ and $D$ be the union of ranges of $E_{L}-E_{-L}$ for all $L>0$. $D$ is a core of $e^{z H}$ for any $z$. For each $\Phi \in D$, we have

$$
(\Psi(z), \Phi)=\left(\Psi, e^{\bar{z} H} \Phi\right)
$$

for pure imaginary $z$. Both sides are holomorphic in $z$ for $\operatorname{Re} z \in(0, \delta)$ and continuous in $z$ for $\operatorname{Re} z \in[0, \delta]$. Hence the equality holds for all $z$ with $\operatorname{Re} z \in[0, \delta]$ by the edge of wedge theorem. Since $D$ is a core of $e^{\bar{z} H}$, the equality holds for all $\Phi \in D\left(e^{\bar{z} H}\right)$. Hence $\Psi \in D\left(e^{z H}\right)$ and $\Psi(z)=$ $e^{z H} \Psi$.

Therefore we obtain Proposition if we show that the right hand side of (4.24) is holomorphic for $\operatorname{Re} z \in(0,1 / 2)$, is strongly continuous for $\operatorname{Re} z \in[0,1 / 2]$, and sequentially strongly continuous in $h$.

Due to (3.2), the sum in (4.24) converges uniformly in norm for $\operatorname{Re} z \in[0,1 / 2]$ and over a bounded set of $h$. Due to Theorem 3.1, the integrand in each term of (4.24) is holomorphic for $\operatorname{Re} z \in(0,1 / 2)$ and continuous for $\operatorname{Re} z \in[0,1 / 2]$. Since the integrand is dominated by $\|h\|^{n}\|\Psi\|$ irrespective of $s_{k}$ and $z$, we obtain the holomorphy of the integral by Fubini's theorem applied to Cauchy integral formula. We also obtain the strong continuity in $z$ by Lebesgue dominated convergence theorem applied to the inner product with other vector and the norm. Hence we have holomorphy and continuity of the sum by the uniform convergence.

Exactly the same proof as Proposition 4.1 shows that the right hand side of (4.24) is sequentially strongly continuous in $h$.

Q.E.D.

Proposition 4.13. Let $h \in \mathfrak{M}, h^{*}=h, \Psi$ be a cyclic and separating 
vector, $\Phi=\Psi(h), \quad \psi=\omega_{\Psi}$ and $\varphi=\omega_{\mathscr{\omega}}$. Assume that $l_{1}^{1 / 2} \Psi \geqq \Phi \geqq l_{2}^{1 / 2} \Psi$ for some $l_{1}$ and $l_{2}$. Then there exists an invertible $\mathrm{A}(z) \in \mathfrak{M}$ for $\operatorname{Re} z \in$ $[0,1 / 2]$ such that $\mathrm{A}(z)$ is holomorphic for $\operatorname{Re} z \in(0,1 / 2)$, strongly continuous for $\operatorname{Re} z \in[0,1 / 2],\|\mathrm{A}(z)\| \leqq \max \left(l_{1}^{1 / 2}, 1\right),\left\|\mathrm{A}(z)^{-1}\right\| \leqq \max \left(l_{2}^{-1 / 2}, 1\right)$, $\mathrm{A}(s+i t)=u_{t}^{\varphi \psi} \sigma_{t}^{\psi}\{A(s)\}, \mathrm{A}(0)=1, \mathrm{~A}(1 / 2) \Psi=\Phi, \Psi(z)=\mathrm{A}(z) \Psi$.

Proof. Consider $\Psi(z)$ of Proposition 4.12. We have

$$
\Psi(s+i t)=\exp i t\left(-H_{\Psi}+h\right) \Psi(s)=u_{t}^{\varphi \psi} \Delta_{\Psi}^{i t} \Psi(s)
$$

due to (4.14). By Theorem 3 (8) of $[2]$, there exists $A \in \mathfrak{M}$ such that $\Phi=A \Psi,\|A\| \leqq l_{1}^{1 / 2},\left\|A^{-1}\right\| \leqq l_{2}^{-1 / 2}$. Hence

$$
\Psi(i t)=u_{t}^{\varphi \psi} \Psi, \Psi(1 / 2+i t)=u_{t}^{\varphi \psi} \sigma_{t}^{\psi}(A) \Psi
$$

For a vector $\chi$ and $Q \in \mathfrak{M}^{\prime}$, consider

$$
f(z)=\left(\Psi(z), Q^{*} x\right)
$$

Since $\|\Psi(z)\| \leqq e^{\|h\|}\|\Psi\|, \mathrm{f}(z)$ is uniformly bounded for $\operatorname{Re} z \in[0,1 / 2]$. By Proposition 4.12, it is holomorphic for $\operatorname{Re} z \in(0,1 / 2)$ and continuous for $\operatorname{Re} z \in[0,1 / 2]$. Furthermore, by (4.27), we have the following bounds on the boundary lines:

$$
\begin{gathered}
|\mathrm{f}(i t)|=\left|\left(u_{t}^{\varphi \psi} \Psi, Q^{*} x\right)\right|=\left|\left(u_{t}^{\varphi \psi} Q \Psi, x\right)\right| \\
\leqq \\
\qquad Q \Psi|\||| x\|, \\
|\mathrm{f}(1 / 2+i t)|=\left|\left(u_{t}^{\varphi \psi} \sigma_{t}^{\psi}(A) \Psi, Q^{*} x\right)\right|=\left|\left(u_{t}^{\varphi \psi} \sigma_{t}^{\psi}(A) Q \Psi, x\right)\right| \\
\leqq \\
\leqq A|||| Q \Psi \mid\|x\| .
\end{gathered}
$$

Hence we have

$$
|\mathrm{f}(z)| \leqq \max (\|A\|, 1)\|Q \Psi|\|\mid x\| .
$$

This implies the existence of operators $A(z)$ such that

$$
\mathrm{f}(z)=(\mathrm{A}(z) Q \Psi, \chi),\|\mathrm{A}(z)\| \leqq \max \left(l_{1}^{1 / 2}, 1\right)
$$

due to Riesz theorem and $\|A\| \leqq l_{1}^{1 / 2}$.

Since $f(z)=(Q \Psi(z), x)$, we have 


$$
Q \Psi(z)=\mathrm{A}(z) Q \Psi, Q \in \mathfrak{M}^{\prime}
$$

Hence $\left[\mathrm{A}(z), Q^{\prime}\right] Q \Psi=0$ for any $Q, Q^{\prime} \in \mathfrak{M}^{\prime}$ and hence $\mathrm{A}(z) \in \mathfrak{M}$.

By interchanging the role of $\Psi$ and $\Phi$, we obtain $\mathrm{B}(z) \in \mathfrak{M}$ such that $\mathrm{B}(i t)=u_{t}^{\psi \varphi}$ and $\|\mathrm{B}(z)\| \leqq \max \left(l_{2}^{-1 / 2}, 1\right)$. Since $\mathrm{A}(i t) \mathrm{B}(i t)=u_{t}^{\varphi t} u_{t}^{\psi \mid \varphi}=1$, we have $\mathrm{A}(z) \mathrm{B}(z)=1$. Similarly $\mathrm{B}(z) \mathrm{A}(z)=1$. Hence $\left\|\mathrm{A}(z)^{-1}\right\|=\|\mathrm{B}(z)\| \leqq$ $\max \left(l_{2}^{-1 / 2}, 1\right)$.

Since $\Psi(z)$ is strongly continuous for $\operatorname{Re} z \in[0,1 / 2]$, we have the strong continuity of $\mathrm{A}(z) Q^{\prime} \Psi=Q^{\prime} \Psi(z)$ for all $Q^{\prime} \in \mathfrak{M}$. Since $\mathrm{A}(z)$ is uniformly bounded, it is strongly continuous for $\operatorname{Re} z \in[0,1 / 2]$.

Since $\Psi(z)$ is holomorphic for $\operatorname{Re} z \in(0,1 / 2)$, we have

$$
\begin{aligned}
\mathrm{A}(z) Q^{\prime} \Psi & =Q^{\prime} \Psi(z)=(2 \pi i)^{-1} \int_{\Gamma}\left(z-z^{\prime}\right)^{-1} Q^{\prime} \Psi\left(z^{\prime}\right) \mathrm{d} z^{\prime} \\
& =\left\{(2 \pi i)^{-1} \int_{\Gamma}\left(z-z^{\prime}\right)^{-1} \mathrm{~A}\left(z^{\prime}\right) \mathrm{d} z^{\prime}\right\} Q^{\prime} \Psi
\end{aligned}
$$

for any $Q^{\prime} \in \mathfrak{M}^{\prime}$ and any simple closed curve $\Gamma$, encircling the point $z$ and contained in the $\operatorname{strp}\{z ; \operatorname{Re} z \in(0,1 / 2)\}$. Hence $\mathrm{A}(z)$ is holomorphic for $\operatorname{Re} z \in(0,1 / 2)$.

The equality $\mathrm{A}(s+i t)=u_{t}^{\varphi \psi} \sigma_{t}^{\dagger}\{\mathrm{A}(s)\}$ follows from (4.26) and $\Psi(z)=$ $\mathrm{A}(z) \Psi$.

Q.E.D.

Proposition 4.14. In Proposition 4.13, $\sigma_{z}^{\psi}(h)$ has an analytic continuation to $\operatorname{Im} z \in(-1 / 2,1 / 2)$.

Proof. Since $\mathrm{A}(z)$ and $\mathrm{A}(z)^{-1}$ is holomorphic in $z$ for $\operatorname{Re} z \in(0,1 / 2)$, the operator

$$
\mathrm{B}(z)=\mathrm{A}(z)^{-1}(\mathrm{~d} / \mathrm{d} z) \mathrm{A}(z)
$$

is also holomorphic for $\operatorname{Re} z \in(0,1 / 2)$. For pure imaginary $z=i t$, we have $\mathrm{A}(z)=u_{t}^{\varphi t}=\operatorname{Exp}_{r}\left(\int_{0}^{t} ; i \sigma_{s}^{\psi}(h) \mathrm{d} s\right)$ and hence

$$
\mathrm{B}(i t)=\sigma_{t}^{t}(h) \text {. }
$$

Since $\mathrm{B}(z)$ is holomorphic for $\operatorname{Re} z \in(0,1 / 2), \mathrm{B}(-\bar{z})^{*}$ is holomorphic for $\operatorname{Re} z \in(-1 / 2,0)$. We also have $\mathrm{B}(i t)=\mathrm{B}(-\overline{i t}) *$. By edge of wedge theorem, there exists an operator valued analytic function $h(z)$ for $\operatorname{Re} z \in$ 
$(-1 / 2,1 / 2)$ such that $\mathrm{h}(z)=\mathrm{B}(z)$ for $\operatorname{Re} z \in(0,1 / 2), \mathrm{h}(z)=\mathrm{B}(-\bar{z})^{*}$ for $\operatorname{Re} z \in(-1 / 2,0)$ and $\mathrm{h}(i t)=\sigma_{t}^{\dagger}(h)$.

Q.E.D.

\section{§5. Existence Proof (I)}

Lemma 5.1. Let $\Phi \in V_{\Psi}$,

$$
\begin{aligned}
& \mathrm{F}(t)=2 \pi \operatorname{sech}^{2} 2 \pi t, \\
& \Phi_{\mathrm{F}}=\int_{-\infty}^{\infty} \Delta_{\Psi}^{i t} \Phi \mathrm{F}(t) \mathrm{d} t .
\end{aligned}
$$

Then $\Phi_{\mathrm{F}}$ is in $D\left(\Delta_{\Psi}^{i z}\right)$ for $\operatorname{Im} z \in(-1 / 4,1 / 4)$, it is in $V_{\Psi}$ and satisfies

$$
\Phi=\int_{-1 / 4}^{1 / 4} \Delta_{T}^{t} \Phi_{\mathrm{F}} \mathrm{d} t
$$

If $\Phi=Q \Psi, Q \in \mathfrak{M}$, then $\Phi_{\mathrm{F}}=Q_{\mathrm{F}} \Psi$ where $\psi=\omega_{\Psi}$ and

$$
Q_{\mathrm{F}}=\int_{-\infty}^{\infty} \sigma_{t}^{\psi}(Q) \mathrm{F}(t) \mathrm{d} t
$$

Proof. We have

$$
\tilde{\mathrm{F}}(u) \equiv \int e^{i u t} \mathrm{~F}(t) \mathrm{d} t=u\left(e^{u / 4}-e^{-u / 4}\right)^{-1}
$$

Hence $\Phi_{\mathrm{F}}=\tilde{\mathrm{F}}\left(\log \Delta_{\Psi}\right) \Phi$ is in the domain of $\Delta_{\Psi}^{i z}$ for $\operatorname{Im} z \in(-1 / 4,1 / 4)$ because $e^{\alpha u} \tilde{\mathrm{F}}(u)$ is bounded for $|\operatorname{Re} \alpha|<1 / 4$. We also have

$$
\int_{-1 / 4}^{1 / 4} \tilde{\mathrm{F}}(u) e^{t u} \mathrm{~d} t=1
$$

Hence (5.3) holds.

Since $V_{\Psi}$ is a convex cone invariant under $\Delta_{\Psi}^{i t}$ and $\mathrm{F}(t)>0$, we have $\Phi_{\mathrm{F}} \in V_{\Psi}$. If $\Phi=Q \Psi$, then (5.4) follows from (5.2) due to $\Delta_{\bar{T}}^{i t} \Psi=\Psi$.

Q.E.D.

Lemma 5.2. Let $Q \in \mathfrak{M}$ be such that $Q \Psi \in V_{\Psi}, \sigma_{t}^{\psi}(Q)$ has an "analytic continuation" $\sigma_{z}^{\psi}(Q) \in \mathfrak{M}$ for $\operatorname{Im} z \in[-\delta, 0]$ and $\left\|\sigma_{-i \delta}(Q)-1\right\| \leqq L$ where $\delta$ is any fixed number in $(0,1 / 8)$. Let $Q_{\mathrm{F}}$ be given by $(5.4)$. Then $\sigma_{t}^{\psi}\left(Q_{\mathrm{F}}\right)$ 
has an "analytic continuation" $\sigma_{z}^{\downarrow}\left(Q_{\mathrm{F}}\right) \in M$ for $\operatorname{Im} z \in(-\delta-1 / 4, \delta+3 / 4)$,

$$
h_{1} \equiv \sigma_{i / 4}^{\psi}\left(Q_{\mathrm{F}}\right)-2=\sigma_{i / 4}^{\psi /}\left\{(Q-1)_{\mathrm{F}}\right\}
$$

is in $\mathfrak{M}, h_{1}^{*}=h_{1},\left\|h_{1}\right\| \leqq 2 L$,

$$
\Psi_{1} \equiv \Psi\left(h_{1}\right)=\operatorname{Exp}_{r}\left(\int_{0}^{1 / 2} ; \sigma \underline{\psi}_{i s}\left(h_{1}\right) \mathrm{d} s\right) \Psi
$$

and

$$
Q_{1} \equiv Q \operatorname{Exp}_{l}\left(\int_{0}^{1 / 2} ;-\sigma \Psi_{i s}\left(h_{1}\right) \mathrm{d} s\right)
$$

satisfy $Q_{1} \Psi_{1}=Q \Psi, \sigma_{z}^{\prime}\left(Q_{1}\right) \in \mathfrak{M}$ for $\operatorname{Im} z \in\left[-\delta_{1}, 0\right]$, and

$$
\begin{aligned}
& \left\|\sigma_{-i \delta_{1}}^{\psi_{1}}\left(Q_{1}\right)-1\right\| \leqq\left(L^{2}+(1+L) L^{\prime}\right) e^{L / 2}, \quad \phi_{1}=\omega_{\Psi_{1}}, \\
& L^{\prime}=(1 / 2)\left\{\pi L \log 2\left(\delta-\delta_{1}\right)\right\}^{2} \exp \left\{-\pi L \log 2\left(\delta-\delta_{1}\right)\right\}
\end{aligned}
$$

where $\delta_{1}$ is any number in $(0, \delta)$.

Proof. By Theorem 3(7) of $[2], Q \Psi \in V_{\Psi}$ implies that $\sigma_{t}^{\text {th }}(Q)$ has an "analytic continuation" $\sigma_{z}^{\text {t) }}(Q) \in \mathfrak{M}$ for $\operatorname{Im} z \in[0,1 / 2]$ and $\left(\sigma_{z}^{\text {中 }}(Q)\right)^{*}=$ $\sigma_{\frac{\psi}{z}+i / 2}(Q)$. ("Analytic continuation" here means a function continuous in the closed strip and holomorphic in the interior.) By assumption, we also have an "analytic continuation" $\sigma_{z}^{\psi}(Q) \in \mathfrak{M}$ for $\operatorname{Im} z \in[-\delta, 0]$. By edge of wedge theorem, we have $\sigma_{z}^{\psi}(Q) \in \mathfrak{M}$ for $\operatorname{Im} z \in[-\delta, 1 / 2+\delta],\left(\sigma_{z}^{\psi}(Q)\right)^{*}$ $=\sigma_{\bar{z}+i / 2}^{\psi}(Q)$ and

$$
\left\|\sigma_{z}^{\psi}(Q-1)\right\| \leqq\left\|\sigma_{i \delta}^{\psi}(Q-1)\right\| \leqq L, \operatorname{Im} z \in[-\delta, 1 / 2+\delta] .
$$

(see proof of Lemma 6 of [2].)

By (5.4), we have an analytic continuation

$$
\sigma_{z}^{\psi}\left(Q_{\mathrm{F}}\right)=\int_{-\infty}^{\infty} \sigma_{t}^{\psi}\left(\sigma_{z_{1}}^{\psi}(Q)\right) \mathrm{F}\left(t-z_{2}\right) \mathrm{d} t \in \mathfrak{M}
$$

whenever $z=z_{1}+z_{2}, \operatorname{Im} z_{1} \in(-\delta, 1 / 2+\delta)$ and $\operatorname{Im} z_{2} \in(-1 / 4,1 / 4)$ i.e. for $\operatorname{Im} z \in(-\delta-1 / 4,3 / 4+\delta)$. Since $Q_{\mathrm{F}} \Psi \in V_{\Psi}$, we have

$$
\sigma_{i / 4}^{\psi}\left(Q_{\mathrm{F}}\right) \geqq 0
$$

by Theorem $3(7)$ of $[2]$. Hence $h_{1}^{*}=h_{1}$. Since $\tilde{\mathrm{F}}(0)=2,1_{\mathrm{F}}=2$ and we 
have the second equality of (5.6). By proof of Lemma 6(4) of [2] and the equality $\sigma_{i / 2}^{\psi}\left(Q_{\mathrm{F}}-2\right)=\left(Q_{\mathrm{F}}-2\right) *$, we have $\left\|h_{1}\right\| \leqq\left\|Q_{\mathrm{F}}-2\right\| \leqq 2\|Q-1\| \leqq$ $2\left\|\sigma \psi_{i \delta}(Q-1)\right\| \leqq 2 L$ where the third inequality follows from proof of Lemma $6(4)$ of $[2]$ and $\left(\sigma_{z}^{\psi}(Q)\right) *=\sigma_{z+i / 2}^{\psi}(Q)$.

Let

$$
Q_{1}^{\prime}=\operatorname{Exp}_{l}\left(\int_{0}^{1 / 2} ;-\sigma_{-i s}\left(h_{1}\right) \mathrm{d} s\right)-1+\int_{0}^{1 / 2} \sigma_{-i s}\left(h_{1}\right) \mathrm{d} s
$$

By Lemma 5.1,

$$
\left(\int_{0}^{1 / 2} \sigma_{-i s}\left(h_{1}\right) \mathrm{d} s\right) \Psi=\int_{-1 / 4}^{1 / 4} \Delta_{\Psi}^{s} Q_{\mathrm{F}} \Psi \mathrm{d} s-\Psi=(Q-1) \Psi
$$

Since $\Psi$ is separating $\int_{0}^{1 / 2} \sigma_{-i s}\left(h_{1}\right) \mathrm{d} s=Q-1$. Hence the definition (5.8) implies

$$
Q_{1}=1-(Q-1)^{2}+Q Q_{1}^{\prime}
$$

We have

$$
\begin{aligned}
& \left\|\sigma_{z}^{\psi}\left(Q_{1}\right)-1\right\| \leqq\left\|\sigma_{z}^{\psi}(Q)-1\right\|^{2}+\left\{1+\left\|\sigma_{z}^{\psi}(Q)-1\right\|\right\}\left\|\sigma_{z}^{\psi}\left(Q_{1}^{\prime}\right)\right\|, \\
& \left\|\sigma_{z}^{\psi}\left(Q_{1}^{\prime}\right)\right\| \leqq \sum_{n=2}^{\infty} \int_{0}^{1 / 2} \mathrm{~d} s_{1} \ldots \int_{0}^{s_{n-1}} \mathrm{~d} s_{n}\left\|\sigma_{z-i s_{1}}^{\psi}\left(h_{1}\right)\right\| \cdots\left\|\sigma_{z-i s_{n}}^{\psi}\left(h_{1}\right)\right\| \\
& =\sum_{n=2}^{\infty}(n !)^{-1}\left\{\int_{0}^{1 / 2} \mathrm{~d} s\left\|\sigma_{z-i s}^{\psi}\left(h_{1}\right)\right\|\right\}^{n} .
\end{aligned}
$$

By (5.10), (5.6) and (5.9), we have

$$
\begin{aligned}
\left\|\sigma_{z-i s}^{\psi}\left(h_{1}\right)\right\| & \leqq \int_{-\infty}^{\infty}\left\|\sigma_{z-i s+i \mathrm{a}(s)+i / 4}^{\psi}(Q-1)\right\||\mathrm{F}(t+i \mathrm{a}(s))| \mathrm{d} t \\
& \leqq L \int_{-\infty}^{\infty}|\mathrm{F}(t+i \mathrm{a}(s))| \mathrm{d} t=8 \pi L \mathrm{a}(s) / \sin 4 \pi \mathrm{a}(s)
\end{aligned}
$$

if $\mathrm{a}(s)$ is chosen such that

$$
\operatorname{Im} z-s+\mathrm{a}(s)+1 / 4 \in[-\delta, 1 / 2],|\mathrm{a}(s)|<1 / 4 .
$$

If $\operatorname{Im} z \in\left[-\delta_{1}, 0\right]$ and $\delta_{1}<\delta$, one can choose

$$
\mathrm{a}(s)=(s-1 / 4)\left(1-2\left(\delta-\delta_{1}\right)\right) .
$$


For $|\mathrm{a}(s)|<1 / 4$, we have $|\mathrm{a}(s)(1 / 4-|\mathrm{a}(s)|) / \sin 4 \pi \mathrm{a}(s)| \leqq 2^{-5}$ due to $|x / \sin x| \leqq \pi / 2$ for $|x| \leqq \pi / 2$ and $\sin (\pi-x)=\sin x$. Hence

$$
\begin{aligned}
\int_{0}^{1 / 2} \mathrm{~d} s\left\|\sigma_{z-i s}^{\prime}\left(h_{1}\right)\right\| & \leqq\left(2-4\left(\delta-\delta_{1}\right)\right)^{-1} \pi L\left|\log 2\left(\delta-\delta_{1}\right)\right| \\
& \leqq \pi L\left|\log 2\left(\delta-\delta_{1}\right)\right|
\end{aligned}
$$

Since

$$
\sum_{n=2}^{\infty}(n !)^{-1} x^{n}=e^{x}-1-x \leqq x^{2} e^{x} / 2, x \geqq 0,
$$

we have

$$
\left\|\sigma_{z}^{\psi}\left(Q_{1}^{\prime}\right)\right\| \leqq L^{\prime}, \operatorname{Im} z \in\left[-\delta_{1}, 0\right]
$$

By analytic continuation of $(4.9), \sigma_{t}^{\prime 1}\left(Q_{1}\right)$ has the following analytic continuation:

$$
\sigma_{z}^{\psi_{1}}\left(Q_{1}\right)=\operatorname{Exp}_{r}\left(\int_{0}^{1} ; i z \sigma_{z s}^{\psi}\left(h_{1}\right) \mathrm{d} s\right) \sigma_{z}^{\psi}\left(Q_{1}\right) \operatorname{Exp}_{l}\left(\int_{0}^{1} ;-i z \sigma_{z s}^{\psi}\left(h_{1}\right) \mathrm{d} s\right) .
$$

By (5.6), (5.9) and $\int F(t) \mathrm{d} t=2$, we have $\left\|\sigma^{w_{i s}}\left(h_{1}\right)\right\| \leqq 2 L$ for $0 \leqq s \leqq \delta_{1}$ and hence $\left\|\sigma_{-i \delta_{1}}^{t_{1}}\left(Q_{1}\right)\right\| \leqq\left\|\sigma_{-i \delta_{1}}\left(Q_{1}\right)\right\| \exp 4 \delta_{1} L \leqq\left(L^{2}+(1+L) L^{\prime}\right) e^{L / 2}$.

Finally, $Q_{1} \Psi_{1}=Q \Psi$ follows from formula (2.15) of [3].

Q.E.D.

Lemma 5.3. Let $Q \in \mathfrak{M}$ be such that $Q \Psi \in V_{\Psi}, \sigma_{z}^{\text {tr }} Q \in \mathfrak{M}$ for $\operatorname{Im} z \in$ $[-\delta, 0], \delta \in(0,1 / 8)$ and $\left\|\sigma_{i \delta} Q-1\right\| \leqq L_{0}$,

$$
L_{0} \leqq(4 \pi \log \delta)^{-2}
$$

Then there exists $h \in \mathfrak{M}, h^{*}=h$ such that $Q \Psi=\Psi(h)$.

Proof. We fix $\delta_{n}=2^{-n} \delta, n=0,1, \ldots$ By Lemma 5.2 , we obtain a sequence of vectors $\Psi_{n}$, operators $Q_{n} \in \mathfrak{M}$, operators $h_{n} \in \mathfrak{M}$ and positive numbers $L_{n}, n=1,2, \ldots$ such that $\Psi_{n}=\Psi_{n-1}\left(h_{n}\right), \Psi_{0}=\Psi, h_{n}^{*}=h_{n},\left\|h_{n}\right\|$ $\leqq 2 L_{n-1}, \omega_{\Psi_{n}}=\psi_{n}, \sigma_{t}^{\psi_{n}} Q_{n}$ has an analytic continuation $\sigma_{z}^{\psi_{n}} Q_{n}$ for $\operatorname{Im} z \in$ $\left[-\delta_{n}, 0\right], \quad\left\|\sigma_{-i \delta_{n}}^{\psi_{n}}\left(Q_{n}\right)-1\right\| \leqq L_{n}, Q_{n} \Psi_{n}=Q \Psi\left(\in V_{\Psi}\right), \quad L_{n}=\left(L_{n-1}^{2}+\left(1+L_{n-1}\right)\right.$ $\left.L_{n-1}^{\prime}\right) e^{L_{n-1} / 2}$ and 


$$
L_{n-1}^{\prime}=(1 / 2)\left\{\pi L_{n-1} \log 2\left(\delta_{n-1}-\delta_{n}\right)\right\}^{2} \exp \left\{-\pi L_{n-1} \log 2\left(\delta_{n-1}-\delta_{n}\right)\right\}
$$

We prove that $L_{n} \leqq 2^{-n} L_{0}$ inductively. Assume that $L_{n-1} \leqq 2^{-(n-1)} L_{0}$. We have

$$
\begin{aligned}
& \left\{\pi \log 2\left(\delta_{n-1}-\delta_{n}\right)\right\}^{2} L_{n-1} \leqq \pi^{2}(\log \delta-(n-1) \log 2)^{2} 2^{-(n-1)} L_{0} \\
& \leqq 2 \pi^{2}(\log \delta)^{2} L_{0}+2 \pi^{2}(\log 2)^{2}(n-1)^{2} 2^{-(n-1)} L_{0}
\end{aligned}
$$

Since $n^{2} 2^{-n} \leqq 9 / 8$, we have $(\log 2)^{2}(n-1)^{2} 2^{-(n-1)} \leqq(\log \delta)^{2}$ and

$$
\left\{\pi \log 2\left(\delta_{n-1}-\delta_{n}\right)\right\}^{2} L_{n-1} \leqq 4 \pi^{2}(\log \delta)^{2} L_{0} \leqq 1 / 4
$$

Hence we also have

$$
\exp \left\{-\pi \log 2\left(\delta_{n-1}-\delta_{n}\right) L_{n-1}\right\} \leqq \exp \left(L_{0}^{1 / 2} / 2\right)
$$

Since $L_{n-1} \leqq L_{0} \leqq 2^{-4}$, we have

$$
\begin{aligned}
L_{n} / L_{n-1} & =L_{n-1}+\left(1+L_{n-1}\right) L_{n-1}^{\prime} / L_{n-1} \\
& \leqq\left(2^{-4}+\left(1+2^{-4}\right)(1 / 8) \exp (1 / 8)\right) e^{1 / 32}<1 / 2 .
\end{aligned}
$$

This proves $L_{n} \leqq 2^{-n} L_{0}$.

Since $\left\|h_{n}\right\| \leqq 2 L_{n-1}, h=\sum h_{n}$ is norm convergent and hence $\lim \Psi_{n}=\Psi(h)$ by Lemmas 4.5 and 4.1. Since $Q_{n} \Psi_{n} \in V_{\Psi}=V_{\Psi_{n}}$, where $\Psi_{n}=\Psi\left(h_{1}+\cdots+h_{n}\right)$ is cyclic and separating by Corollary $4.4, \sigma_{t}^{\prime \prime} n\left(Q_{n}\right)$ has an analytic continuation $\sigma_{z}^{\psi_{n}}\left(Q_{n}\right)$ for $\operatorname{Im} z \in(0,1 / 2)$ and $\left(\sigma_{z}^{\psi_{n}}\left(Q_{n}\right)\right)^{*}=\sigma_{z+i / 2}^{\psi_{n}}\left(Q_{n}\right)$. Hence we have the analytic continuation $\sigma_{z}^{t_{n}}\left(Q_{n}\right)$ for $\operatorname{Im} z \in\left[-\delta_{n}, 1 / 2+\delta_{n}\right]$ and by proof of Lemma 6 of [2],

$$
\left\|Q_{n}-1\right\| \leqq\left\|\sigma_{-i \delta_{n}}^{\phi_{n}}\left(Q_{n}\right)-1\right\| \leqq L_{n} .
$$

Hence $\lim \left\|Q_{n}-1\right\|=0$ and we have

$$
Q \Psi=\lim Q_{n} \Psi_{n}=\Psi(h)
$$

Q.E.D.

Proposition 5.4. Let $Q \in \mathfrak{M}$ be such that $J_{\Psi} Q \Psi=Q \Psi$ and $\sigma_{t}^{\phi}(Q)$ has an "analytic continuation" $\sigma_{z}^{\psi} Q$ for $\operatorname{Im} z \in[-1 / 2,0]$. Then there exists $h \in \mathfrak{M}$ such that $h^{*}=h$ and $e^{Q} \Psi=\Psi(h)$. 
Proof. By assumption, $Q \Psi=J_{\Psi} Q \Psi$ which implies $\Delta_{\Psi}^{-1 / 2} Q \Psi=Q^{*} \Psi$. Hence $\sigma_{t}^{\text {t }}(Q)$ has an "analytic continuation" $\sigma_{z}^{\text {t) }}(Q)$ for $\operatorname{Im} z \in[0,1 / 2]$ by Lemma 6 of $[2]$. It satisfies $\sigma_{i / 2}^{\psi}(Q)=Q^{*}$, which implies $\sigma_{z}^{\psi}(Q)^{*}=$ $\sigma_{z+i / 2}^{\psi}(Q)$ for $\operatorname{Im} z=0$ and hence for $\operatorname{Im} z \in[-1 / 2,0]$ by an "analytic continuation". In particular

$$
\sigma_{i / 4}^{\psi}(Q)^{*}=\sigma_{i / 4}^{\psi}(Q)
$$

Let $\Phi_{t}=e^{t Q \Psi}, \varphi_{t}=\omega_{\Phi_{t}}$. By Theorem 3 (7) of $[2], \sigma_{i / 4}^{\phi}\left(e^{t Q}\right)=\exp$ $t \sigma_{i / 4}^{\psi}(Q) \geqq 0$ implies $\Phi_{t} \in V_{\Psi}$ for real $t$.

By Lemma 7 of $[2]$, we have

$$
\Delta_{\Phi_{t}}^{1 / 2} Q \Phi_{t}=e^{t Q} \sigma_{\underline{i}_{i 2}}(Q) e^{-t Q} \Phi_{t}
$$

By Lemma 6 of $[2], \sigma_{z}^{\varphi_{t}}(Q)$ has an "analytic continuation" for $\operatorname{Im} z \in$ $[-1 / 2,0]$ and

$$
\left\|\sigma_{z}^{\varphi} t(Q)\right\| \leqq a=\max \left\{\|Q\|, e^{2 t\|Q\|}\left\|\sigma_{-i / 2}^{\psi}(Q)\right\|\right\}
$$

We now choose $N$ such that

$$
\exp (a / N)-1 \leqq(4 \log \delta)^{-2}
$$

for a fixed $\delta \in(0,1 / 8)$. Then

$$
\begin{aligned}
\left\|\sigma_{-i \delta}^{\varphi_{t}}\left(e^{Q / N}-1\right)\right\| & =\left\|\exp \left\{\sigma_{-i \delta}^{\varphi_{t}}(Q) / N\right\}-1\right\| \\
& \leqq \exp \left\{\left\|\sigma_{-i \delta}^{\varphi_{t}}(Q)\right\| / N\right\}-1 \leqq(4 \log \delta)^{-2}
\end{aligned}
$$

We can now apply Lemma 5.3 and find $h_{n} \in \mathfrak{M}$ for each integer $n \in[0, N]$ such that $\Phi_{n / N}=\Phi_{(n-1) / N}\left(h_{n}\right), h_{n}^{*}=h_{n}$. Then

$$
e^{Q} \Psi=\Phi_{N / N}=\Psi\left(\sum_{n=1}^{N} h_{n}\right)
$$

Q.E.D.

Remark. Vectors $e^{Q} \Psi$ satisfying the condition of Proposition 5.4 are dense in $V_{\Psi}$, which can be seen as follows.

The vectors $\Delta_{\Psi}^{1 / 4} x \Psi, x \in \mathfrak{M}, x \geqq 0$ are dense in $V_{\Psi}$ by definition. Furthermore 


$$
\left\|\Delta^{1 / 4}\left(x-x^{\prime}\right) \Psi\right\|^{2} \leqq 2\left\|\left(x-x^{\prime}\right) \Psi\right\|^{2}
$$

for $x-x^{\prime}=\left(x-x^{\prime}\right) * \in \mathfrak{M}$ by equation (3.13) of [2]. Let $x=\int \lambda \mathrm{d} E_{\lambda}, x_{L}=$ $x\left(E_{L}-E_{1 / L}\right)+(1 / L) E_{1 / L}+\left(1-E_{L}\right), y_{L, \beta}=\left(\log x_{L}\right)\left(f_{\beta}^{G}\right)$. Then

$$
\lim _{L \rightarrow \infty} \lim _{\beta \rightarrow+0} e^{y_{L, \beta} \Psi}=x \Psi
$$

and hence $Q_{L, \beta}=\sigma_{-i / 4} y_{L, \beta}$ satisfy

$$
\lim _{L \rightarrow \infty} \lim _{\beta \rightarrow+0} e^{Q_{L, \beta}} \Psi=\Delta \mathbb{\Psi}^{1 / 4} x \Psi
$$

$\sigma_{z}^{\psi} Q_{L, \beta}$ is an entire function of $z$ and $J_{\Psi} Q_{L, \beta} \Psi=Q_{L, \beta} \Psi$ due to $\sigma_{i / 4}^{\prime \prime}\left(Q_{L, \beta}\right)$ $=y_{L, \beta}=y_{L, \beta}^{*}$. Hence $Q_{L, \beta}$ satisfies the requirement for $Q$ in Proposition 5.4 .

\section{§6. Existence Proof (II)}

We use the technique introducted by Connes. (See [5].)

Lemma 6.1. Let $\Psi$ be a cyclic and separating vector for a von Neumann algebra $\mathfrak{M}$ on a Hilbert space $\mathfrak{S}$ and $h \in \mathfrak{M}, h^{*}=h \neq 0$. Let $\mathfrak{N}$ be a type $I_{2}$ factor on 4 dimensional space $\Re,\left\{u_{i j}\right\}$ and $\left\{u_{i j}^{\prime}\right\}$ be matrix units of $\mathfrak{R}$ and $\mathfrak{N}^{\prime}$, and $\left\{e_{i j}\right\}$ be an orthonormal basis of $\mathfrak{R}$ such that $u_{i j} e_{k l}=\delta_{j k} e_{i l}$ and $u_{i j}^{\prime} e_{k l}=\delta_{j l} e_{k i}$. Let $\hat{\mathfrak{M}}=\mathfrak{M} \otimes \mathfrak{N}, 0<\lambda<1$ and

$$
x_{h, \lambda}=\lambda^{1 / 2} \Psi \otimes e_{11}+(1-\lambda)^{1 / 2} \Psi(h) \otimes e_{22} .
$$

Then $x_{h, \lambda}$ is a cyclic and separating vector of $\hat{\mathfrak{M}}$, the modular conjugation operator for $x_{h, \lambda}$ is $J_{\Psi} \otimes J_{e}, e=e_{11}+e_{22}$, and the modular operator $\Delta$ for $\chi_{h, \lambda}$ is given by

$$
\begin{aligned}
& \Delta\left(\sum_{i j} \Phi_{i j} \otimes e_{i j}\right)=\sum_{i j}\left(\Delta_{i j} \Phi_{i j}\right) \otimes e_{i j}, \\
& \Delta_{11}=\Delta_{\Psi}, \Delta_{22}=\Delta_{\Psi(h)}, \\
& \Delta_{12}=\{\lambda /(1-\lambda)\} \exp \left(-H_{\Psi}-j(h)\right), \\
& \Delta_{21}=\{(1-\lambda) / \lambda\} \exp \left(-H_{\Psi}+h\right) .
\end{aligned}
$$


Proof. Any $Q \in \hat{\mathfrak{M}}$ can be decomposed as $Q=\sum Q_{i j} u_{i j}, \quad Q_{i j}=$ $\sum_{k} u_{k i} Q u_{j k} \in \mathfrak{M} \otimes 1$. For $J=J_{\Psi} \otimes J_{e}$, we have

$$
\begin{aligned}
& \left(x_{h, \lambda}, Q j(Q) x_{h, \lambda}\right) \\
& =\lambda\left(\Psi, Q_{11} j_{\Psi}\left(Q_{11}\right) \Psi\right)+\lambda^{1 / 2}(1-\lambda)^{1 / 2}\left(\Psi, Q_{12} j_{\Psi}\left(Q_{12}\right) \Psi(h)\right) \\
& +\lambda^{1 / 2}(1-\lambda)^{1 / 2}\left(\Psi(h), Q_{21} j_{\Psi}\left(Q_{21}\right) \Psi\right)+(1-\lambda)\left(\Psi(h), Q_{22} j_{\Psi}\left(Q_{22}\right) \Psi(h)\right)
\end{aligned}
$$

Due to $\Psi \in V_{\Psi}$ and $\Psi(h) \in V_{\Psi}$, the right hand side is positive. Since $J=$ $J_{\Psi} \otimes J_{e}$ obviously satisfies $J \hat{\mathfrak{M}} \mathcal{C} J=\hat{\mathfrak{M}}^{\prime}\left(=\mathfrak{M}^{\prime} \otimes \mathfrak{N}^{\prime}\right), \quad J x_{h, \lambda}=x_{h, \lambda}$ and is an antiunitary involution, it is the modular conjugation operator for $x_{h, \lambda}$ by Theorem 1 of $[2]$.

Since $\omega_{\chi_{h, \lambda}}\left(\left(1 \otimes u_{j j}\right) Q\right)=\omega_{\chi_{h, \lambda}}\left(Q\left(1 \otimes u_{j j}\right)\right), 1 \otimes u_{j j}$ commutes with $\Delta$. Hence $1 \otimes j_{e}\left(u_{k k}\right)$ also commutes with $\Delta$. Since $u_{j j} j_{e}\left(u_{k k}\right) e_{i l}=\delta_{i j} \delta_{k l} e_{j k}$, we have

$$
\Delta^{1 / 2} \sum_{i j} \Phi_{i j} \otimes e_{i j}=\sum_{i j} \Delta_{i j}^{1 / 2} \Phi_{i j} \otimes e_{i j}
$$

For $Q=\Sigma Q_{i j} u_{i j}$ and $\Phi=Q x_{h, \lambda}=\Sigma \Phi_{i j} \otimes e_{i j}$, we have $\Delta^{1 / 2} Q x_{h, \lambda}=J Q^{*} \chi_{h, \lambda}$. Hence

$$
\begin{aligned}
& \Delta_{11}^{1 / 2} Q_{11} \Psi=J_{\Psi} Q_{11}^{*} \Psi=\Delta_{\Psi}^{1 / 2} Q_{11} \Psi, \\
& \Delta_{22}^{1 / 2} Q_{22} \Psi(h)=J_{\Psi} Q_{22}^{*} \Psi(h)=\Delta_{\Psi}^{1 / 2}(h) Q_{22} \Psi(h), \\
& \Delta_{12}^{1 / 2} Q_{12} \Psi(h)=J_{\Psi} Q_{12}^{*}(1-\lambda)^{-1 / 2} \lambda^{1 / 2} \Psi, \\
& \Delta_{21}^{1 / 2} Q_{21} \Psi=J_{\Psi} Q_{21}^{*} \lambda^{-1 / 2}(1-\lambda)^{1 / 2} \Psi(h) .
\end{aligned}
$$

Since $\hat{\mathfrak{M}} \mathrm{x}_{h, \lambda}$ is a core of $\Delta^{1 / 2}, Q_{i j} \Psi_{j}\left(\Psi_{1}=\Psi, \Psi_{2}=\Psi(h)\right)$ must be a core of $\Delta_{i j}^{1 / 2}$. (6.6) implies that two selfadjoint operators $\Delta_{11}^{1 / 2}$ and $\Delta_{\Psi}^{1 / 2}$ coincide on their core and hence must be equal. Similarly (6.7) implies $\Delta_{22}^{1 / 2}=\Delta_{\Psi}^{1 / 2}(h)$. Hence we have (6.3).

To prove (6.4) and (6.5), we first consider the case where $h$ is replaced by $h_{\beta}=h\left(f_{\beta}^{G}\right)$. Then $\Psi\left(h_{\beta}\right)=A \Psi=j(A) \Psi, \Psi=A^{-1} \Psi\left(h_{\beta}\right)=$ $j\left(A^{-1}\right) \Psi\left(h_{\beta}\right)$ where $A \in \mathfrak{M}, A^{-1} \in \mathfrak{M}$ and $A=\operatorname{Exp}_{r}\left(\int_{0}^{1 / 2} ; \sigma_{-i s}\left(h_{\beta}\right) \mathrm{d} s\right)$. We have 


$$
\begin{aligned}
J_{\Psi} Q_{12}^{*} \Psi & =\Delta_{\Psi}^{1 / 2} Q_{12} \Psi=\Delta_{\Psi}^{1 / 2} Q_{12} j_{\Psi}\left(A^{-1}\right) \Psi\left(h_{\beta}\right) \\
& =\Delta_{\Psi}^{1 / 2} j_{\Psi}\left(A^{-1}\right) Q_{12} \Psi\left(h_{\beta}\right)=j_{\Psi}\left(\Delta_{\Psi}^{-1 / 2} A^{-1}\right) Q_{12} \Psi\left(h_{\beta}\right)
\end{aligned}
$$

The inverse $A^{-1}=\operatorname{Exp}_{l}\left(\int_{0}^{1 / 2} ;-\sigma_{-i s}^{\psi}\left(h_{\beta}\right) \mathrm{d} s\right)$ is an analytic continuation of

$$
\begin{array}{r}
u_{t}^{\phi \varphi}=\left(u_{t}^{\varphi \psi}\right)^{*}=\operatorname{Exp}_{l}\left(\int_{0}^{t} ;-i \sigma_{s}^{\dagger}\left(h_{\beta}\right) \mathrm{d} s\right) \\
=\operatorname{Exp}_{l}\left(\int_{0}^{1 / 2} ;-i(2 t) \sigma_{2 t s}^{\dagger}\left(h_{\beta}\right) \mathrm{d} s\right)
\end{array}
$$

to $t=-i / 2$, where $\varphi=\omega_{\Psi\left(h_{\beta}\right)}$. By an analytic continuation of (4.15), we obtain

$$
\Delta_{\bar{\Psi}}^{-1 / 2} A^{-1}=\exp \left(\left(H_{\Psi}-h_{\beta}\right) / 2\right)
$$

which holds on $A \mathrm{D}\left(\Delta_{\bar{\Psi}}^{-1 / 2}\right)$. (See second paragraph of proof of Proposition 4.12.) Hence we have

$$
\begin{gathered}
\Delta_{12}^{1 / 2} Q_{12} \Psi\left(h_{\beta}\right)=(\lambda /(1-\lambda))^{1 / 2} j_{\Psi}\left\{\exp \left(\left(H_{\Psi}-h_{\beta}\right) / 2\right)\right\} Q_{12} \Psi\left(h_{\beta}\right) \\
=(\lambda /(1-\lambda))^{1 / 2} \exp \left(-\left(H_{\Psi}+j\left(h_{\beta}\right)\right) / 2\right) Q_{12} \Psi\left(h_{\beta}\right) .
\end{gathered}
$$

Since $\mathfrak{M} \Psi(h)$ is a core of $\Delta_{12}^{1 / 2}$ and both $\Delta_{12}^{1 / 2}$ and $\exp \left(-\left(H_{\Psi}+j\left(h_{\beta}\right)\right) / 2\right)$ are selfadjoint, we have (6.4) for $h_{\beta}$.

Hence

$$
\begin{aligned}
\Delta_{12}^{i t} & =(\lambda /(1-\lambda))^{i t} \exp \left(-i t\left(H_{\Psi}+j\left(h_{\beta}\right)\right)\right) \\
& =(\lambda /(1-\lambda))^{i t} j\left\{\exp \left(-i t\left(H_{\Psi}-h_{\beta}\right)\right)\right\} \\
& =(\lambda /(1-\lambda))^{i t} j\left\{u_{t}^{\varphi \phi} \Delta_{\Psi}^{i t}\right\} .
\end{aligned}
$$

As $\beta \rightarrow+0, \Delta^{i t}$ for $\chi_{h_{\beta}, \lambda}$ tends strongly to $\Delta^{i t}$ for $\chi_{h, \lambda}$ by Theorem 10 of [2], and $u_{t}^{\varphi \phi}$ for $\varphi=\omega_{\Psi\left(h_{\beta}\right)}$ tends strongly to $u_{t}^{\varphi \psi}$ for $\varphi=\omega_{\Psi(h)}$. Hence we have (6.4) for a general $h$.

From (6.9), we have

$$
\Delta_{21}^{1 / 2} Q_{21} \Psi=((1-\lambda) / \lambda)^{1 / 2} \Delta_{\Psi}^{1 / 2} A^{*} Q_{21} \Psi .
$$

Since $A^{*}=\operatorname{Exp}_{l}\left(\int_{0}^{1 / 2} ; \sigma_{i s}^{\phi}\left(h_{\beta}\right) \mathrm{d} s\right)$ is an analytic continuation of $(6.10)$ to 
$t=i / 2$, we obtain as before

$$
\Delta_{\Psi}^{1 / 2} A^{*}=\exp \left(-\left(H_{\Psi}-h_{\beta}\right) / 2\right)
$$

By taking the limit $\beta \rightarrow+0$, we obtain (6.5).

Q.E.D.

Remark. If $u^{\prime}$ is a unitary operator in $\mathfrak{M}^{\prime}$ and

$$
\chi=\lambda^{1 / 2} \Psi \otimes e_{11}+(1-\lambda)^{1 / 2} u^{\prime} \Psi(h) \otimes e_{22},
$$

then

$$
\hat{u}^{\prime}=1 \otimes j\left(u_{11}\right)+u^{\prime} \otimes j\left(u_{22}\right)
$$

is a unitary element of $\hat{\mathfrak{M}}^{\prime}$ and $x=\hat{u}^{\prime} x_{h, \lambda}$. Hence modular conjugation operator and modular operator for such $\chi$ are given by $\hat{u}^{\prime}\left(J_{\Psi} \otimes J_{e}\right)\left(\hat{u}^{\prime}\right)^{*}$ and $\hat{u}^{\prime} \Delta\left(\hat{u}^{\prime}\right) *$.

Lemma 6.2. If $h_{n} \in \mathfrak{M}, h_{n}^{*}=h_{n}, \lim \Psi\left(h_{n}\right)=\Phi$ (strongly), $\Phi$ is cyclic and separating and $l_{1}^{1 / 2} \Psi \geqq \Psi\left(h_{n}\right) \geqq l_{2}^{1 / 2} \Psi$ for strictly positive $l_{1}$ and $l_{2}$ independent of $n$, then $h=\mathrm{w}-\lim h_{n}$ exists and $\Phi=\Psi(h)$.

Proof. Let $\chi_{n} \equiv \chi_{h_{n}, \lambda}$ be defind as (6.1) and

$$
\chi=\lambda^{1 / 2} \Psi \otimes e_{11}+(1-\lambda)^{1 / 2} \Phi \otimes e_{22}
$$

Then $\Phi=\lim \Psi\left(h_{n}\right)$ implies $\chi=\lim \chi_{n} . \quad \chi$ is cyclic and separating and by Theorem 10 of $[2]$

$$
\lim _{n} \Delta_{\chi_{n}}^{i t}=\Delta_{\chi}^{i t}, \lim _{n} \Delta_{\Psi\left(h_{n}\right)}^{i t}=\Delta_{\emptyset}^{i t}
$$

where the convergence is in the strong operator topology and is uniform over a compact set of $t$.

Since $\left(\Delta_{\chi_{n}}\right)_{21}^{i t} \otimes u_{22} j\left(u_{11}\right)=\Delta_{\chi_{n}}^{i t}\left(1 \otimes u_{22} j\left(u_{11}\right)\right)$, and $\left(\Delta_{\chi}\right)_{21}^{i t} \otimes u_{22} j\left(u_{11}\right)$ $=\Delta_{\chi}^{i t}\left(1 \otimes u_{22} j\left(u_{11}\right)\right)$, we have

$$
\lim _{n} \exp i t\left(-H_{\Psi}+h_{n}\right)=\exp i t\left(\log \left(\Delta_{\chi}\right)_{21}\right)
$$

by (6.5) where the convergence is uniform in $t$ over a compact set.

By multiplying $e^{-t}$ and integrating over $t \in[0, \infty)$, we obtain 


$$
\lim _{n}\left\{\left(-H_{T}+h_{n}\right)+i\right\}^{-1}=\left\{\log \left(\Delta_{\chi}\right)_{21}+i\right\}^{-1}
$$

Hence, by subtracting $\left(-H_{\Psi}+i\right)^{-1}$, we have

$$
\begin{aligned}
\lim ( & \left.-H_{\Psi}+i\right)^{-1} h_{n}\left\{\left(-H_{\Psi}+h_{n}\right)+i\right\}^{-1} \\
& =\left(-H_{\Psi}+i\right)^{-1}-\left\{\log \left(\Delta_{\chi}\right)_{21}+i\right\}^{-1} .
\end{aligned}
$$

By Corollary $4.11,\left\|h_{n}\right\|$ is uniformly bounded. Hence we obtain

$$
\left\|\left(-H_{\Psi}+i\right)^{-1} h_{n}\left[\left\{\left(-H_{\Psi}+h_{n}\right)+i\right\}^{-1}-\left\{\log \left(\Delta_{\chi}\right)_{21}+i\right\}^{-1}\right] \xi\right\| \rightarrow 0 .
$$

Hence $\left(-H_{\Psi}+i\right)^{-1} h_{n} \eta$ is strongly convergent for any $\eta$ in the range of $\left\{\log \Delta_{21}+i\right\}^{-1}$, which is a dense set. Since $\left\|\left(-H_{\Psi}+i\right)^{-1} h_{n}\right\|$ is uniformly bounded, we have the existence of

$$
\lim _{n}\left(-H_{\Psi}+i\right)^{-1} h_{n} \equiv h_{0}
$$

For $\xi_{2} \in \mathrm{D}\left(\left(-H_{\Psi}+i\right)^{*}\right)$, we have

$$
\left|\left(h_{0} \xi_{1},\left(-H_{\Psi}+i\right)^{*} \xi_{2}\right)\right|=\lim _{n}\left|\left(h_{n} \xi_{1}, \xi_{2}\right)\right| \leqq \sup || h_{n}|||| \xi_{1}|||| \xi_{2} \| .
$$

Hence $h_{0} \xi_{1} \in \mathrm{D}\left(\left(-H_{\Psi}+i\right)\right)$ and $\left\|\left(-H_{\Psi}+i\right) h_{0}\right\| \leqq \sup \left\|h_{n}\right\|$. We have

$$
\mathrm{w}-\lim h_{n}=h \equiv\left(-H_{\Psi}+i\right) h_{0} .
$$

By (4.14), we have

$$
u_{t}^{\varphi_{n}{ }^{4}}=\left\{\exp i t\left(-H_{\Psi}+h_{n}\right)\right\} \Delta_{\Psi}^{i t},
$$

which is strongly convergent, uniformly in $t$ over a compact set, due to (6.12), where $\varphi_{n} \equiv \omega_{\Psi\left(h_{n}\right)}$.

By Proposition 4.13, there exists $\mathrm{A}_{n}(z) \in \mathfrak{M}$ for $\operatorname{Re} z \in[0,1 / 2]$ such that $\mathrm{A}_{n}(z)$ is holomorphic for $\operatorname{Re} z \in(0,1 / 2)$, strongly continuous for $\operatorname{Re} z \in[0,1 / 2],\left\|\mathrm{A}_{n}(z)\right\| \leqq \max \left(l_{1}^{1 / 2}, 1\right),\left\|\mathrm{A}_{n}(z)^{-1}\right\| \leqq \max \left(l_{2}^{-1 / 2}, 1\right), \mathrm{A}_{n}(s+i t)$ $=u_{t}^{\varphi}{ }^{\psi} \sigma_{t}^{\psi}\left\{\mathrm{A}_{n}(s)\right\}, \mathrm{A}_{n}(0)=1$ and $\mathrm{A}_{n}(1 / 2) \Psi=\Psi\left(h_{n}\right)$.

Since $\mathrm{A}_{n}(i t) Q^{\prime} \Psi=Q^{\prime}\left\{\exp i t\left(-H_{\Psi}+h_{n}\right)\right\} \Psi$ and $A_{n}((1 / 2)+i t) Q^{\prime} \Psi=$ $Q^{\prime} u_{t}^{\varphi}{ }^{\phi} \sigma_{t}^{\psi}\left\{A_{n}(1 / 2)\right\} \Psi=Q^{\prime}\left\{\exp i t\left(-H_{\Psi}+h_{n}\right)\right\} \Psi\left(h_{n}\right)$ for $Q^{\prime} \in \mathfrak{M}^{\prime}$ are strongly convergent, uniformly over real $t$ in a compact set, and since $\| A_{n}((1 / 2)$ $+i t) \|$ and $\left\|A_{n}(i t)\right\|$ are uniformly bounded, $A_{n}((1 / 2)+i t)$ and $A_{n}(i t)$ are 
strongly convergent, uniformly over real $t$ in a compact set.

Since $\mathrm{A}_{n}(z)$ is holomorphic for $\operatorname{Re} z \in(0,1 / 2)$ and is continuous and uniformly bounded for $\operatorname{Re} z \in[0,1 / 2]$, we have

$$
\begin{aligned}
e^{z^{2}} \mathrm{~A}_{n}(z) & =(2 \pi)^{-1} \int_{-\infty}^{\infty}(z-i t)^{-1} e^{-t^{2}} \mathrm{~A}_{n}(i t) \mathrm{d} t \\
& -(2 \pi)^{-1} \int_{-\infty}^{\infty}(z-i t-1 / 2)^{-1} e^{-(t-i / 2)^{2}} \mathrm{~A}_{n}(i t+1 / 2) \mathrm{d} t
\end{aligned}
$$

for $\operatorname{Re} z \in(0,1 / 2)$. Hence

$$
\begin{aligned}
& (\mathrm{d} / \mathrm{d} z) e^{z^{2}} \mathrm{~A}_{n}(z) \\
= & (2 \pi)^{-1} \int_{-\infty}^{\infty}(z-i t-1 / 2)^{-2} e^{-(t-i / 2)^{2}} \mathrm{~A}_{n}(i t+1 / 2) \mathrm{d} t \\
- & (2 \pi)^{-1} \int_{-\infty}^{\infty}(z-i t)^{-2} e^{-t^{2}} \mathrm{~A}_{n}(i t) \mathrm{d} t .
\end{aligned}
$$

Since $\left\|\mathrm{A}_{n}(i t)\right\|$ and $\left\|\mathrm{A}_{n}(i t+1 / 2)\right\|$ are uniformly bounded in $t$ and $n$, the integral converges uniformly in $n$. Hence (6.15) is strongly convergent as $n \rightarrow \infty$, uniformly in $z$ over any compact subset of $\{z ; \operatorname{Re} z \in(0,1 / 2)\}$.

By $l_{1}^{1 / 2} \Psi \geqq \Psi\left(h_{n}\right) \geqq l_{2}^{1 / 2} \Psi$ and $\Phi=\lim \Psi\left(h_{n}\right)$, we have $l_{1}^{1 / 2} \Psi \geqq \Phi \geqq l_{2}^{1 / 2} \Psi$. By Theorem $3(8)$ of $[2]$, there exists $A \in \mathfrak{M}$ such that $A \Psi=\Phi,\|A\| \leqq l_{1}^{1 / 2}$ and $\left\|A^{-1}\right\| \leqq l_{2}^{-1 / 2}$. Then $A=\lim _{n} \mathrm{~A}_{n}(1 / 2)$.

For $Q^{\prime} \in \mathfrak{M I}^{\prime}$ we have

$$
\begin{aligned}
& \left\|\left(\sigma_{t}^{\varphi_{n}}\left\{\mathrm{~A}_{n}(1 / 2)^{-1}\right\}-\sigma_{t}^{\varphi}\left\{A^{-1}\right\}\right) Q^{\prime} \Phi\right\| \\
& =\| Q^{\prime}\left(\sigma_{t}^{\varphi_{n}}\left\{\mathrm{~A}_{n}(1 / 2)^{-1}\right\} \Psi\left(h_{n}\right)-\sigma_{t}^{\varphi}\left\{A^{-1}\right\} \Phi\right) \\
& \quad-Q^{\prime} \sigma_{t}^{\varphi_{n}}\left\{\mathrm{~A}_{n}(1 / 2)^{-1}\right\}\left(\Psi\left(h_{n}\right)-\Phi\right) \| \\
& \leqq\left\|Q^{\prime}\right\|\left\|\left(\Delta_{\Psi\left(h_{n}\right)}^{i t}-\Delta_{\Phi}^{i t}\right) \Psi\right\|+\left\|Q^{\prime} \mid\right\| l_{2}^{-1 / 2}\left\|\Psi\left(h_{n}\right)-\Phi\right\|
\end{aligned}
$$

which converges to 0 as $n \rightarrow \infty$ uniformly in $t$ over a compact set due to $\lim \Psi\left(h_{n}\right)=\Phi$ and Theorem 10 of $[2]$, where $\varphi=\omega_{\varpi}$. Hence

$$
\lim _{n} \sigma_{t}^{\varphi_{n}}\left\{\mathrm{~A}_{n}(1 / 2)^{-1}\right\}=\sigma_{t}^{\varphi}\left\{A^{-1}\right\}
$$

uniformly in $t$ over a compact set. 
By (6.11),

$$
u_{t}^{\phi \varphi_{n}}=\Delta^{i t}\left(\Delta_{\chi_{n}}\right)_{2 i}^{-i t}\{\lambda /(1-\lambda)\}^{-i t}
$$

is strongly convergent, uniformly over a compact set of $t$.

Since $\left\|\mathrm{A}_{n}(z)^{-1}\right\|$ is uniformly bounded, the analyticity and the continuity of $A_{n}(z)$ imply the same properties for $A_{n}(z)^{-1}$. Hence

$$
\mathrm{A}_{n}(s+i t)^{-1}=\sigma_{t}^{\psi}\left(\mathrm{A}_{n}(s)^{-1}\right) u_{t}^{\psi \varphi_{n}}=u_{t}^{\psi \varphi_{n}} \sigma_{t}^{\varphi_{n}}\left(\mathrm{~A}_{n}(s)^{-1}\right)
$$

is strongly convergent as $n \rightarrow \infty$, for $s \in[0,1 / 2]$, which is proved in exactly the same way as before by use of the Cauchy integral formula of the form (6.14).

Combining the convergences of (6.15) and (6.16), we have the strong convergence of

$$
\mathrm{A}_{n}(z)^{-1}(\mathrm{~d} / \mathrm{d} z)\left\{e^{z^{2}} \mathrm{~A}_{n}(z)\right\} \equiv \mathrm{F}_{n}(z),
$$

as $n \rightarrow \infty$ uniformly in $z$ over any compact subset of $\{z ; \operatorname{Re} z \in(0,1 / 2)\}$.

By Theorem 1 of [3] and (4.6), we have

$$
(\mathrm{d} / \mathrm{d} t) u_{t}^{\varphi_{n} \psi}=i u_{t}^{\varphi_{n} \phi} \sigma_{t}^{\phi}\left(h_{n}\right) .
$$

Hence

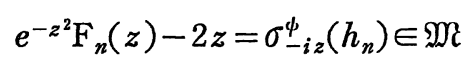

for $z=i$. Hence

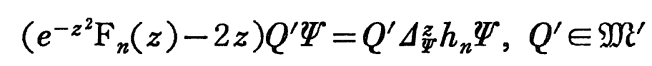

which holds for all $z$ satisfying $\operatorname{Re} z \in[0,1 / 2]$. Since $\mathrm{w}-\lim h_{n}=h$ and $\mathrm{F}_{n}(z)$ has a strong limit, we have

$$
\lim _{n}\left(e^{-z^{2}} \mathrm{~F}_{n}(z)-2 z\right) Q^{\prime} \Psi=Q^{\prime} \Delta_{\Psi}^{z} h \Psi
$$

for $Q^{\prime} \in \mathfrak{N}_{T_{2}}$ (see $\S 3$ of $[2]$ for the definition of $\mathfrak{N}_{\Psi 2}$ ) and $\operatorname{Re} z \in(0,1 / 2$ ). Since $\lim \mathrm{F}_{n}(z) \in \mathfrak{M}$, this implies the existence of an analytic continuation $\sigma_{z}^{\psi}(h)$ of $\sigma_{t}^{\psi}(h)$ to $z$ in $\{z ; \operatorname{Re} z \in(0,1 / 2)\}$.

By (6.15) and the uniform boundedness of $\mathrm{A}_{n}(z)$, we obtain the uniform boundedness of $\sigma_{z}^{\psi}\left(h_{n}\right)$ over any compact subset of $\{z ; \operatorname{Re} z \in(0$, 
$1 / 2)\}$ and over all $n$. Hence $\lim _{n} \sigma_{z}^{\phi}\left(h_{n}\right)=\sigma_{z}^{\phi}(h)$ and

$$
\begin{aligned}
\Delta_{\Psi}^{t_{m}} h_{n} \Delta_{\Psi}^{t_{m}} h_{n} & \ldots \Delta_{\Psi}^{t_{1}} h_{n} \Psi \\
& =\sigma_{-}^{\Psi_{i t_{m}}}\left(h_{n}\right) \ldots \sigma_{-i\left(t_{m}+\cdots+t_{1}\right)}\left(h_{n}\right) \Psi
\end{aligned}
$$

is strongly convergent as $n \rightarrow \infty$ for $t_{j}>0, t_{1}+\cdots+t_{m}<1 / 2$.

Since $\left\|h_{n}\right\|$ is uniformly bounded by $\max \left(\left|\log l_{1}\right|,\left|\log l_{2}\right|\right)$, we have

$$
\lim _{n} \Psi\left(h_{n}\right)=\Psi(h)
$$

by Lebesgue dominated convergence Theorem (for inner product with other vectors and for its norm). Hence $\Phi=\Psi(h)$.

Q.E.D.

Theorem 6.3. If $l_{1} \geqq l_{2}>0$ and $l_{1}^{1 / 2} \Psi \geqq \Phi \geqq l_{2}^{1 / 2} \Psi$ for cyclic and separating $\Psi$ and $\Phi$, then there exists $h \in \mathfrak{M}$ such that $h^{*}=h, \Phi=\Psi(h)$ and $\log l_{1} \geqq h \geqq \log l_{2}$.

Remark. If $\varphi$ and $\psi$ are normal faithful states satisfying $l_{1} \psi \geqq \varphi \geqq l_{2} \psi$, then the unique representative $\xi_{\varphi}$ and $\xi_{\psi}$ in a fixed canonical cone $V_{\boldsymbol{\Psi}}$ satisfies $l_{1}^{1 / 2} \xi_{\psi} \geqq \xi_{\varphi} \geqq l_{2}^{1 / 2} \xi_{\psi}$ by Theorem $3(8)$ and (9) of [2] and hence there exists $h \in \mathfrak{M}$ such that $h^{*}=h, \Phi=\Psi(h)$ and $\log l_{1} \geqq h \geqq \log l_{2}$ due to this theorem.

Proof. By Theorem 3(8) of $[2]$ and the assumption $l_{1}^{1 / 2} \Psi \geqq \Phi \geqq l_{2}^{1 / 2} \Psi$, there exists $Q \in \mathfrak{M}$ such that $\Phi=Q \Psi$. By Theorem 3(7) of [2] and $l_{1}^{1 / 2} \Psi \geqq Q \Psi \geqq l_{2}^{1 / 2} \Psi,\left(\Delta_{\Psi}^{i z} Q \Delta_{\Psi}^{-i z}\right)^{-} \in \mathfrak{M}$ for $\operatorname{Im} z \in[0,1 / 2]$ and

$$
l_{1}^{1 / 2} \geqq\left(\Delta_{\bar{\Psi}}^{-1 / 4} Q \Delta_{\Psi^{1 / 4}}^{1 /-} \geqq l_{2}^{1 / 2}\right.
$$

where the bar indicates the closure. Let

$$
k \equiv \log \left(\Delta_{\Psi}^{-1 / 4} Q \Delta_{\Psi}^{1 / 4}\right)^{-} .
$$

Then $k \in \mathfrak{M}$ and $\log l_{1} \geqq 2 k \geqq \log l_{2}$. We have

$$
\Phi=Q \Psi=\Delta_{\Psi}^{1 / 4}\left(\Delta_{\Psi}^{-1 / 4} Q \Delta_{\Psi}^{1 / 4}\right) \Psi=\Delta_{\Psi}^{1 / 4} e^{k} \Psi
$$

Let $k_{\beta} \equiv \int \sigma_{t}^{\psi}(k) f_{\beta}^{G}(t) \mathrm{d} t$ where $f_{\beta}^{G}$ is given by (3.11) of [2]. Then 
$k_{\beta}^{*}=k_{\beta},\left\|k_{\beta}\right\| \leqq\|k\|_{i}$ and $\lim _{\beta \rightarrow 0} k_{\beta}=k$. Hence

$$
\left\|\Delta_{\Psi}^{1 / 4}\left(e^{k_{\beta}}-e^{k}\right) \Psi\right\|^{2} \leqq 2\left\|\left(e^{k_{\beta}}-e^{k}\right) \Psi\right\|^{2} \rightarrow 0
$$

by (3.13) of $[2]$ and $\Delta_{\Psi}^{1 / 2}\left(e^{k_{\beta}}-e^{k}\right) \Psi=J_{\Psi}\left(e^{k_{\beta}}-e^{k}\right) \Psi$. Let

$$
\Phi_{\beta}=\Delta_{\Psi}^{1 / 4} e^{k_{\beta}} \Psi
$$

We have $\Phi_{\beta} \in V_{\Psi}$ and $\lim \Phi_{\beta}=\Phi$.

Since $\int f_{\beta}^{G}(t) \mathrm{d} t=1$ and $f_{\beta}^{G}(t) \geqq 0$, we have

$$
\log l_{1} \geqq 2 k_{\beta} \geqq \log l_{2}, \quad l_{1}^{1 / 2} \geqq e^{k_{\beta}} \geqq l_{2}^{1 / 2} .
$$

By Theorem 3(7) of [2], we have

$$
l_{1}^{1 / 2} \Psi \geqq \Phi_{\beta} \geqq l_{2}^{1 / 2} \Psi \text {. }
$$

Since

$$
\sigma_{t}^{\phi}\left(\exp k_{\beta}\right)=\exp \sigma_{t}^{\phi}\left(k_{\beta}\right)
$$

and $\sigma_{t}^{t}\left(k_{\beta}\right)$ has an analytic continuation to an entire function

$$
\sigma_{z}^{\psi}\left(k_{\beta}\right)=\int \sigma_{t}^{\psi}(k) f_{\beta}^{G}(t-z) \mathrm{d} t \in \mathfrak{M}
$$

$\left(\Delta_{\Psi}^{i t} e^{k_{\beta}} \Delta_{\bar{T}}^{-i t}\right.$ ) has an analytic continuation to an entire function $\exp \sigma_{z}^{\top}\left(k_{\beta}\right)$. Hence

$$
\Phi_{\beta}=\left(\Delta_{\Psi}^{1 / 4} e^{h_{\beta}} \Delta_{\bar{\Psi}}^{-1 / 4}\right) \Psi=\exp \left(\sigma \underline{\Psi}_{i / 4}\left(k_{\beta}\right)\right) \Psi
$$

Since $Q_{1} \equiv \sigma_{-i / 4}^{\psi}\left(k_{\beta}\right)$ satisfies $J_{\Psi} Q_{1} \Psi\left(=\Delta_{\Psi}^{1 / 2} Q_{1}^{*} \Psi\right)=Q_{1} \Psi$ and has the property that $\sigma_{t}^{\psi}\left(Q_{1}\right)=\sigma_{t-i / 4}^{\psi}\left(k_{\beta}\right)$ has an analytic continuation to an entire function $\sigma_{z}^{\psi}\left(Q_{1}\right)=\sigma_{z-i / 4}^{\phi}\left(k_{\beta}\right)$, Proposition 5.4 is applicable and there exists $h_{\beta} \in \mathfrak{M}$ such that $\Phi_{\beta}=\Psi\left(h_{\beta}\right), h_{\beta}^{*}=h_{\beta}$. Lemma 6.2 then implies Theorem.

Q.E.D.

Remark. The above proof implies that if $\Phi=\Delta_{\Psi}^{1 / 4} e^{k} \Psi, k=k^{*} \in \mathfrak{M}$, and $\log l_{2} \leqq 2 k \leqq \log l_{1}$, then there exists $h \in \mathfrak{M}, h^{*}=h$ such that $\Phi=\Psi(h)$, and $\log l_{2} \leqq h \leqq \log l_{1}$. 


\section{References}

[1] Araki, H., Publ. RIMS 4(1968), 361-371.

[2] Araki, H., Pacific J. Math., to be published.

[3] Araki, H., Ann. Sci. École Norm. Sup., 6 (1973), 67-84.

[4] Araki, H., Rep. Math. Phys. 4 (1973), 227-254.

[5] Connes, A., C. R. Acad. Sci. Paris A274. (1972), 1923-1926.

Note added in proof: Theorem 3(8) of [2] has been misused in proofs of Propositions 4.13, 4.14, Lemma 6.2 and Theorem 6.3. Hence we need the assumption $l_{1}^{1 / 2} \omega_{\Psi} \geqq \omega_{\emptyset} \geqq l_{2}^{1 / 2} \omega_{\Psi}$ in Propositions 4.13 and 4.14. However, Lemma 6.2 and Theorem 6.3 hold without modification.

For Lemma 6.2, we modify its proof after (6.13) as follows: Since $h-h_{n}$ is weakly convergent to 0 and $\left(-H_{\Psi}+i+h\right)^{-1}$ is strongly convergent,

$$
\begin{aligned}
\left(-H_{\Psi}+i+h_{n}\right)^{-1} & -\left(-H_{\Psi}+i+h\right)^{-1} \\
& =\left(-H_{\Psi}+i+h\right)^{-1}\left(h-h_{n}\right)\left(-H_{\Psi}+i+h_{n}\right)^{-1}
\end{aligned}
$$

is weakly convergent to 0 . By $\left(6.12^{\prime}\right),\left(\Delta_{\chi}\right)_{21}=\exp \left(-H_{\Psi}+h\right)$, which implies that $\Phi$ and $\Psi(h)$ have the same modular automorphisms. Hence $\Phi=e^{\alpha} \Psi(h)$ for a selfadjoint $\alpha$ affiliated with the center. Then $\left(\Delta_{\chi}\right)_{21}$ is calculated to be $\exp \left(-H_{\Psi}+h+\alpha\right)$. Hence $\alpha=0$ and $\Phi=\Psi(h)$.

For Theorem $6.3, \quad l_{1}^{1 / 2} \Psi \geqq \Phi \geqq l_{2}^{1 / 2} \Psi$ directly implies $\Phi=\Delta^{1 / 4} e^{k} \Psi$ for a $k \in \mathfrak{M}$ and $\log l_{1} \geqq 2 k \geqq \log l_{2}$. 
